\title{
RAISING NILPOTENT ORBITS IN WAVE-FRONT SETS
}

\author{
DIHUA JIANG, BAIYING LIU, AND GORDAN SAVIN
}

\begin{abstract}
We study wave-front sets of representations of reductive groups over global or non-archimedean local fields.
\end{abstract}

\section{INTRODUCTION}

Let $k$ be a global or non-archimedean local field. Let $G(k)$, or simply $G$, be the group of $k$-points of a reductive algebraic group defined over $k$, or a central extension of finite degree. Let $\mathfrak{g}$ be the Lie algebra of $G$ and $u$ a nilpotent element in $\mathfrak{g}$. We assume that the characteristic of $k$ is large, so that the Jacobson-Morozov theorem holds, i.e., there exists a homomorphism $\varphi: \mathfrak{s l}_{2} \rightarrow \mathfrak{g}$ such that

$$
u=\varphi\left(\begin{array}{ll}
0 & 0 \\
1 & 0
\end{array}\right) .
$$

(A more detailed discussion on restrictions in the positive characteristic case is in Section 5.) Now assume that $k$ is a local field. Fix a non-trivial character $\psi$ of $k$. Then, to $u$, one can attach a unipotent subgroup $N_{u} \subseteq G$ and a character $\psi_{u}$ of $N_{u}$ (see Section 5 for definitions). For example, if $G$ is $k$-quasisplit and $u$ belongs to a regular orbit, then $N_{u}$ is a maximal unipotent subgroup and $\psi_{u}$ is a Whittaker character. Let $\pi$ be a smooth representation of $G$, not necessarily admissible. The wave-front set of $\pi$ is the set of nilpotent orbits $\mathcal{O}$ such that the space of twisted coinvariants $\pi_{N_{u}, \psi_{u}}$ is non-trivial for $u \in \mathcal{O}$. Our main result concerns the structure of orbits in the wave-front set. Let $\mathfrak{c}$ be the centralizer of $\varphi\left(\mathfrak{s l}_{2}\right)$ in $\mathfrak{g}$. Pick a homomorphism $\varphi_{\mathfrak{c}}: \mathfrak{s l}_{2} \rightarrow \mathfrak{c}$, and let $\mathcal{O}^{\prime}$ be the nilpotent orbit corresponding to $\varphi^{\prime}=\varphi \oplus \varphi_{\mathfrak{c}}$. If $\mathcal{O}$ (corresponding to $\varphi$ ) is contained in the wave-front set of $\pi$, then, under certain conditions, $\mathcal{O}^{\prime}$ is also contained in the wave-front set of $\pi$. Precise statements are contained in Section 6, in particular, Proposition 6.1 and the remark following Corollary 6.4.

Assume, for example, that $G$ is a classical group and $\mathcal{O}$ corresponds to a partition $p$. Then the conditions are automatically satisfied if $p$ is not special, in the sense of Lusztig and Spaltenstein. The larger orbit $\mathcal{O}^{\prime}$ corresponds to a partition $\underline{p}^{\prime}$ obtained from $p$ by replacing a pair $(i, i)$ in $p$ by $(i+1, i-1)$. This process can be continued until we arrive to a nilpotent element whose corresponding partition is special, more precisely, the special expansion $p^{G}$ of $p$. In particular, the maximal orbits in the wave-front set, with respect to the closure ordering, are special. This result, if $k$ has characteristic zero and odd residual characteristic, was previously obtained by Mœglin in [Mo96] for irreducible representations using the relationship of degenerate Whittaker models and the character expansion of representations

Received by the editors May 4, 2016 and, in revised form, October 6, 2016.

2010 Mathematics Subject Classification. Primary 11F70, 22E50; Secondary 11F85, 22 E55.

Key words and phrases. Nilpotent orbits, wave-front sets, representations, automorphic forms. 
obtained in the work of Mœglin and Waldspurger in [MW87]. The restriction on the residual characteristic has been removed by Varma V14.

Our method works equally well in the global setting. Let $k$ be a global field and $\mathbb{A}$ the corresponding ring of adèles. Let $\psi$ be a non-trivial character of $\mathbb{A}$, trivial on $k$. Let $\Pi$ be a space of smooth functions on $G(k) \backslash G(\mathbb{A})$ stable under the action of $G(\mathbb{A})$ by right translations. Every nilpotent element $u$ in $\mathfrak{g}$ defines a character $\psi_{u}$ of $N_{u}(k) \backslash N_{u}(\mathbb{A})$ and a functional on $\Pi$ by

$$
\int_{N_{u}(k) \backslash N_{u}(\mathbb{A})} f(n) \bar{\psi}_{u}(n) d n
$$

where $f \in \Pi$. The set of nilpotent $G(k)$-orbits such that this functional is non-trivial is a (global) wave-front set of $\Pi$. Let $\mathcal{O}$ be an orbit contained in the wave-front set of $\Pi$. Proposition 6.5 states that, under the same conditions as in the local case, a slightly larger orbit $\mathcal{O}^{\prime}$ is also contained in the wave-front set of $\Pi$. As a consequence, if $G$ is classical, only special orbits are maximal in the wave-front set of $\Pi$. For background on the global result the reader can consult GRS03, G06] or JL15. In fact, the basic idea of this paper is already contained in these papers.

Finally, we consider split exceptional groups. In this case there are certain nonspecial orbits that cannot be eliminated, as maximal elements in the wave-front set, by either our or Mœglin's method. More precisely, we show that the maximal orbits in the wave-front set are completely odd orbits, confirming a conjecture of Nevins [N02]. For classical groups completely odd orbits coincide with special orbits but, in general, completely odd orbits form a larger class. The first example of a completely odd but non-special orbit is the minimal orbit for the exceptional group $\mathrm{G}_{2}$. However, this orbit cannot be a maximal element in the wave-front set by a more elaborate argument contained in [LS08. Thus, it is still reasonable to expect that, for algebraic groups, only special orbits appear as the maximal elements in the wave-front set of a representation of $G$.

\section{Heisenberg GRoup}

Assume, from now on, that the characteristic of $k$ is not 2. Let $\mathfrak{h}$ be a Heisenberg Lie algebra over $k$ with the center $\mathfrak{z}$. Let $H=\exp (\mathfrak{h})$ be the Heisenberg group. As a set, $H=\mathfrak{h}$, but the multiplication in $H$ is given by the Campbell-Hausdorff formula,

$$
x \cdot y=x+y+\frac{1}{2}[x, y]
$$

where $x, y \in H=\mathfrak{h}$ and $[x, y]$ is the Lie bracket. In this way the exponential map is the identity map on the underlying sets. The center of $H$ is $Z=\exp (\mathfrak{z})$. A polarization of $H$ is a decomposition of the Lie algebra

$$
\mathfrak{h}=\log (X) \oplus \log (Y) \oplus \mathfrak{z}
$$

such that $X=\exp (\log X)$ and $Y=\exp (\log Y)$ are abelian subgroups of $H$. The group $X Z$ is a maximal abelian subgroup of $H$.

Assume now that $k$ is a local field. Fix a non-trivial character $\psi$ of $k$. By choosing an identification $Z$ with $k$ we view $\psi$ as a character of $Z$. For every $y \in Y$ we have a character $\psi_{y}: X \rightarrow \mathbb{C}^{\times}$given by

$$
\psi_{y}(x)=\psi([y, x]) \text { for every } x \in X
$$


where $[x, y]=x y x^{-1} y^{-1}$, the group commutator of $x$ and $y$. Let $\rho_{\psi}$ be the representation of $H$ obtained by the smooth induction of the character $1 \otimes \psi$ of $X Z$. The representation $\rho_{\psi}$ is realized on the space $S(Y)$ of Schwartz functions on $Y$. The action on $f \in S(Y)$ is given by

$$
\rho_{\psi}(y)(f)(u)=f(u y) \text { for every } y \in Y
$$

and

$$
\rho_{\psi}(x)(f)(u)=\psi_{u}(x) f(u) \text { for all } x \in X .
$$

The same formulae also define the action of $H$ on $L^{2}(Y)$. This is the Schrödinger model of the unique irreducible unitary representation of $H$ with the central character $\psi$. The subspace of $H$-smooth vectors in $L^{2}(Y)$ is $S(Y)$.

Proposition 2.1. Let $k$ be a non-archimedean field. Let $\pi$ be a smooth representation of $H$ such that $Z$ acts on $\pi$ as the character $\psi$. The bilinear map $\operatorname{Hom}_{H}\left(\rho_{\psi}, \pi\right) \times \rho_{\psi} \rightarrow \pi$ given by $(A, v) \mapsto A(v)$ descends to a canonical isomorphism

$$
\operatorname{Hom}_{H}\left(\rho_{\psi}, \pi\right) \otimes \rho_{\psi} \cong \pi .
$$

If $\operatorname{Hom}_{H}\left(\rho_{\psi}, \pi\right) \neq 0$, then $\pi_{X, \psi_{y}} \neq 0$ for every $y \in Y$.

Proof. The first part, the isomorphism given by $A \otimes v \mapsto A(v)$, is in Wei03. If $\ell$ is an arbitrary functional on $\operatorname{Hom}_{H}\left(\rho_{\psi}, \pi\right)$ and $\ell_{y}$ is the functional on $S(Y)$ given by evaluating functions $f \in S(Y)$ at $y$, then $\ell \otimes \ell_{y}$ transforms under the action of $X$ as $\psi_{y}$. This proves the second part.

Now assume that $k$ is a global field. Let $k_{v}$ denote either a local non-archimedean completion of $k$, or $k_{\infty}=k \otimes_{\mathbb{Q}} \mathbb{R}$. Let $\mathbb{A}$ be the corresponding ring of adèles. It is a restricted product of all $k_{v}$. Let $\psi$ be an additive character of $\mathbb{A}$ trivial on $k$. As in the local case, every $y \in Y(\mathbb{A})$ defines a character $\psi_{y}$ of $X(\mathbb{A})$. If $y \in Y(k)$, then the character $\psi_{y}$ is trivial on $X(k)$. The group $H(\mathbb{A})$ has an irreducible unitary representation with the central character $\psi$. This representation is unique up to isomorphism. It is realized on $L^{2}(Y(\mathbb{A}))$. The subspace of $H(\mathbb{A})$-smooth vectors in this realization is $S(Y(\mathbb{A}))$. This space is isomorphic to the restricted tensor product of $S\left(Y\left(k_{v}\right)\right)$.

The unique irreducible representation of $H(\mathbb{A})$ has another realization on $L_{\psi}^{2}(H(k) \backslash H(\mathbb{A}))$, where the subscript $\psi$ denotes the subspace of functions transforming as $\psi$ under the action of $Z(\mathbb{A})$. This is the lattice model. In this realization, the subspace $\Theta_{\psi}$ of $H(\mathbb{A})$-smooth vectors is the space of smooth functions on $H(k) \backslash H(\mathbb{A})$ transforming as $\psi$ under the action of $Z(\mathbb{A})$. For every $\theta \in \Theta_{\psi}$ and $u \in Y(\mathbb{A})$, let

$$
f_{\theta}(u)=\int_{X(k) \backslash X(\mathbb{A})} \theta(x u) d x .
$$

Then $\theta \mapsto f_{\theta}$ is an isomorphism of $\Theta_{\psi}$ and $S(Y(\mathbb{A}))$, the spaces of $H(\mathbb{A})$-smooth vectors in the two models of the Heisenberg representation.

Proposition 2.2. Let $k$ be a global field. Let $\Pi$ be a non-zero subspace of $\Theta_{\psi}$, stable under the action of $H(\mathbb{A})$ by the right translation. Then, for every $y \in Y(k)$, there exists $\theta \in \Pi$ such that

$$
\int_{X(k) \backslash X(\mathbb{A})} \theta(x) \bar{\psi}_{y}(x) d x \neq 0 .
$$


Proof. Since the map $\theta \mapsto f_{\theta}$ is injective, there exist $u^{\prime} \in Y(\mathbb{A})$ and $\theta^{\prime} \in \Pi$ such that $f_{\theta^{\prime}}\left(u^{\prime}\right) \neq 0$. Let $\theta$ be the right translate of $\theta^{\prime}$ by $y^{-1} u^{\prime}$. Then

$$
\int_{X(k) \backslash X(\mathbb{A})} \theta(x y) d x \neq 0 .
$$

Next, we have the easy sequence of equalities

$$
\theta(x y)=\theta([x, y] y x)=\theta(y x) \bar{\psi}_{y}(x)=\theta(x) \bar{\psi}_{y}(x)
$$

where, for example, the last identity holds since $\theta$ is left $H(k)$-invariant. Replacing $\theta(x y)$ by $\theta(x) \bar{\psi}_{y}(x)$ in the integral yields the proposition.

\section{JACOBI GROUP}

In this section we introduce a type of Jacobi group that we shall need. A Jacobi group $J$ is a semi-direct product of $M$, the universal central extension of $\mathrm{SL}_{2}$, and the Heisenberg group $H$. (In practice we shall only need finite degree extensions of $\mathrm{SL}_{2}$, however, since the degree is not fixed we are using the universal central extension M.) Let $\mathfrak{s l}_{2}$ be the Lie algebra of $\mathrm{SL}_{2}$. Let $\mathfrak{n}$ and $\overline{\mathfrak{n}}$ be the nilpotent subalgebras of $\mathfrak{s l}_{2}$, consisting of upper and lower triangular matrices respectively. Let $N=\exp (\mathfrak{n})$ and $\bar{N}=\exp (\overline{\mathfrak{n}})$ be the corresponding subgroups of $\mathrm{SL}_{2}$. Then $N$ and $\bar{N}$ canonically lift in $M$. In fact, by a result of Steinberg, the group $M$ is generated by $N$ and $\bar{N}$ satisfying certain relations. The Lie algebra of the linear quotient of $J$ is $\mathfrak{s l}_{2} \oplus \mathfrak{h}$. We assume that, under the adjoint action of $\mathfrak{s l}_{2}$, the Lie algebra $\mathfrak{h}$ of $H$ decomposes as $m V_{2} \oplus \mathfrak{z}$, where $V_{2}$ is the irreducible 2-dimensional representation of $\mathfrak{s l}_{2}$. Let

$$
s=\left(\begin{array}{cc}
1 & 0 \\
0 & -1
\end{array}\right) .
$$

The assumption implies that there is a polarization (2.1) of $\mathfrak{h}$ where $\log X$ and $\log Y$ are the eigenspaces for $s$ with eigenvalues 1 and -1 , respectively. In particular, $\log X \oplus \mathfrak{z}$ is a maximal abelian subalgebra of $\mathfrak{h}$. Since $\mathfrak{n}$ consists of elements in $\mathfrak{s l}_{2}$ of $s$-eigenvalue 2 , the space

$$
\mathfrak{u}=\mathfrak{n} \oplus \log X \oplus \mathfrak{z}
$$

is an abelian subalgebra of the Lie algebra $\mathfrak{s l}_{2} \oplus \mathfrak{h}$. Let $U=\exp (\mathfrak{u})=N X Z$, which we consider as a subgroup of $J$. In order to discuss characters of $U$, it will be convenient to work with an explicit realization of $J$.

Let $e_{0}, \ldots, e_{m}, f_{m}, \ldots, f_{0}$ be a basis of a vector space of dimension $2 m+2$. Let $\langle$,$\rangle be a symplectic form such that$

$$
\left\langle e_{i}, e_{j}\right\rangle=0,\left\langle f_{i}, f_{j}\right\rangle=0 \text { and }\left\langle e_{i}, f_{j}\right\rangle=\delta_{i j} .
$$

Let $\mathrm{Sp}_{2 m+2}$ be the group of linear transformations preserving the form $\langle$,$\rangle , and$ $\mathfrak{s p}_{2 m+2}$ the corresponding Lie algebra. If $A$ is a square matrix, let $A^{\top}$ denote the transpose of $A$ with respect to the opposite diagonal. The Lie algebra $\mathfrak{s p}_{2 m+2}$, in the basis $e_{0}, \ldots, e_{m}, f_{m}, \ldots, f_{0}$, consists of block matrices

$$
\left(\begin{array}{ll}
A & B \\
C & D
\end{array}\right)
$$

where $B=B^{\top}, C=C^{\top}$ and $D=-A^{\top}$. In this identification, the Killing form on the Lie algebra is given by the trace pairing.

In this setting the Heisenberg group $H$ is the unipotent radical of the maximal parabolic subgroup $P$ of $\mathrm{Sp}_{2 m+2}$ stabilizing the line through $e_{0}$. A Levi factor of 
$L$ of $P$ is given as the subgroup of all elements in $P$ stabilizing the line through $f_{0}$. The derived group of $L$ is $\mathrm{Sp}_{2 m}$, the subgroup of $\mathrm{Sp}_{2 m+2}$ fixing $e_{0}$ and $f_{0}$. The conjugation action of $\mathrm{Sp}_{2 m}$ on $H$ gives an isomorphism of $\mathrm{Sp}_{2 m}$ and the group of outer automorphisms of $H$. In order to write $J$, we need to choose an embedding of $\mathrm{SL}_{2}$ into $\mathrm{Sp}_{2 m}$. Roughly speaking, we embed $\mathrm{SL}_{2}$ diagonally into $m$ long-root $\mathrm{SL}_{2}$ 's. Up to conjugation, all such embeddings can be described as follows.

Fix an $m$-tuple $\left(a_{1}, \ldots, a_{m}\right)$ of non-zero elements in $k^{\times}$. Let $\mathrm{SL}_{2}$ be the subgroup of $\mathrm{Sp}_{2 m}$ such that, for every $i \neq 0$, it acts on the plane spanned by $e_{i}, f_{i}$ in the standard way with respect to the basis $a_{i} e_{i}, f_{i}$. We let $J$ be the semi-direct product of $H$ with $M$, the central extension of this $\mathrm{SL}_{2}$.

The algebra $\mathfrak{u}=\mathfrak{n} \oplus \log X \oplus \mathfrak{z}$ now consists of all matrices such that $A=C=$ $D=0$ and (in the case $m=2$ )

$$
B=\left(\begin{array}{ccc}
x_{2} & x_{1} & z \\
0 & n_{1} & x_{1} \\
n_{2} & 0 & x_{2}
\end{array}\right)
$$

where $n_{i}=a_{i} n$ for some $n \in k$. Thus, we identify $\mathfrak{u}$ with triples $(n, x, z)$ where $x=\left(x_{1}, \ldots, x_{m}\right)$. Using the Killing form on $\mathfrak{s p}_{2 m+2}$ the dual space of $\mathfrak{u}$ is identified with the quotient $\mathfrak{s p}_{2 m+2} / \mathfrak{u}^{\perp}$. As a complement of $\mathfrak{u}^{\perp}$ in $\mathfrak{s p}_{2 m+2}$ we can take the set of all matrices such that $A=B=D=0$ and (in the case $m=2$ )

$$
C=\left(\begin{array}{ccc}
x_{2}^{*} & 0 & n_{2}^{*} \\
x_{1}^{*} & n_{1}^{*} & 0 \\
z^{*} & x_{1}^{*} & x_{2}^{*}
\end{array}\right)
$$

where $n_{i}^{*}=a_{i}^{-1} n^{*}$ for some $n^{*} \in k$. Thus, we identify the dual $\mathfrak{u}^{*}$ with the set of triples $\left(n^{*}, x^{*}, z^{*}\right)$ where $x=\left(x_{1}^{*}, \ldots, x_{m}^{*}\right)$. Now the natural pairing $\langle\cdot, \cdot\rangle$ between $\mathfrak{u}$ and $\mathfrak{u}^{*}$ is explicitly given by

$$
\left\langle(n, x, z),\left(n^{*}, x^{*}, z^{*}\right)\right\rangle=\operatorname{tr}(B C)=z z^{*}+2 \sum_{i=1}^{m} x_{i} x_{i}^{*}+m n n^{*} .
$$

If $\psi$ is a non-trivial character of $k$, then any $u^{*} \in \mathfrak{u}$ defines a character $\psi_{u^{*}}$ of $U$ by

$$
\psi_{u^{*}}(\exp (u))=\psi\left(\left\langle u, u^{*}\right\rangle\right) \text {. }
$$

The commutative subalgebra $\log Y$ consists of matrices such that $B=C=0$ and (in the case $m=2$ )

$$
A=\left(\begin{array}{ccc}
0 & y_{1} & y_{2} \\
0 & 0 & 0 \\
0 & 0 & 0
\end{array}\right)
$$

Thus, any element in $\log Y$ is identified with an $m$-tuple $y=\left(y_{1}, \ldots, y_{m}\right)$.

\section{FOURIER-JACOBI MODELS}

In this section we define local and global Fourier-Jacobi models of representations of the Jacobi group $J$ with non-trivial action of the center $Z$. The Fourier-Jacobi model is a representation of $M$.

Fix a non-trivial character $\psi$ of $k$. Realize $J$ as a subgroup of $\operatorname{Sp}_{2 m+2}(k)$, as in the previous section. In particular, we have fixed an identification of $Z$ and $k$. In this way, $\psi$ can be viewed as a character of $Z$, and every $u^{*} \in \mathfrak{u}$ gives a character $\psi_{u^{*}}$ of $U$. 
Assume now that $k$ is a local field. Then, following A. Weil, the representation $\rho_{\psi}$ of the Heisenberg group $H$ extends to $J$. Recall that $M$ is a universal central extension of $\mathrm{SL}_{2}(k)$. We shall need the explicit formula for the action of $N$, the subgroup of $M$ isomorphic to the group of upper triangular unipotent matrices in $\mathrm{SL}_{2}(k)$ under the projection of $M$ onto $\mathrm{SL}_{2}(k)$. We assume that $n \in N$ corresponds to $\left(\begin{array}{ll}1 & x \\ 0 & 1\end{array}\right)$ in $\mathrm{SL}_{2}(k)$. Then for every $f \in S(Y)$ and $y=\exp \left(y_{1}, \ldots, y_{m}\right) \in Y$,

$$
\left(\rho_{\psi}(n) f\right)(y)=\psi\left(x \sum_{i=1}^{m} a_{i} y_{i}^{2}\right) f(y)
$$

where the $a_{i}$ 's are the structure constants of $J$ as in Section 3 (see BS98, Sections 5.1 and 7.2 ] for action formulae).

Let $\pi$ be a smooth representation of $J$ such that the center $Z$ of $H$ acts on $\pi$ by $\psi$. Then there is a representation $\sigma$ of $J$ on $F J_{\psi}(\pi):=\operatorname{Hom}_{H}\left(\rho_{\psi}, \pi\right)$ defined by

$$
A \mapsto \sigma(g)(A)=\pi(g) \cdot A \cdot \rho_{\psi}\left(g^{-1}\right)
$$

for every $g \in J$ and $A \in \operatorname{Hom}_{H}\left(\rho_{\psi}, \pi\right)$. Now the isomorphism in Proposition 2.1 is an isomorphism of $J$-modules. Note that the subgroup $H$ acts trivially on $F J_{\psi}(\pi)$. Hence $\left(\sigma, F J_{\psi}(\pi)\right)$ is a representation of $M$.

Proposition 4.1. Let $k$ be a non-archimedean field. Let $\pi$ be a smooth $J$-module such that $Z$ acts on $\pi$ by the character $\psi$. If $\left(\sigma, F J_{\psi}(\pi)\right)$ is a Whittaker-generic representation of $M$, then $\pi_{U, \psi_{u^{*}}} \neq 0$ for $u^{*}=\left(n^{*}, 0,1\right)$ for some $n^{*} \in k^{\times}$.

Proof. Since $\operatorname{Hom}_{H}\left(\rho_{\psi}, \pi\right)$ is Whittaker-generic, as a representation of $M$, there exist $n^{*} \in k^{\times}$and a non-zero functional $\ell$ on $\operatorname{Hom}_{H}\left(\rho_{\psi}, \pi\right)$ such that

$$
\ell(\sigma(n) A)=\psi\left(n^{*} x\right) \ell(A)
$$

for all $A \in \operatorname{Hom}_{H}\left(\rho_{\psi}, \pi\right)$ and $n \in N$ where $n$ corresponds to $\left(\begin{array}{ll}1 & x \\ 0 & 1\end{array}\right)$. Recall that we have a decomposition

where $\rho_{\psi}$ acts on $S(Y)$. Let

$$
\pi=\sigma \otimes \rho_{\psi}
$$

$$
\ell_{1}: S(Y) \rightarrow \mathbb{C}
$$

be the functional given by evaluating functions at $1 \in Y$. Then $\ell \otimes \ell_{1}$ is a functional of $\pi$ that transforms as $\psi_{u^{*}}$ under the action of $U$ where $u^{*}=\left(n^{*}, 0,1\right)$.

Let $k$ be a global field, and fix a non-trivial character $\psi$ of $Z(\mathbb{A}) / Z(k)$. Let $\rho_{\bar{\psi}}$ (note the complex conjugate!) be the Weil representation of $J(\mathbb{A})$ on $S(Y(\mathbb{A})$ ). For any function $\phi \in S(Y(\mathbb{A}))$ define a theta series

$$
\theta_{\bar{\psi}}^{\phi}(h g)=\sum_{\xi \in Y(k)} \rho_{\bar{\psi}}(h g) \phi(\xi)=\sum_{\xi \in Y(k)} \rho_{\bar{\psi}}(\xi h g) \phi(1)
$$

where $h \in H(\mathbb{A}), g \in M(\mathbb{A})$ and 1 is the identity element of $Y$.

Let $\Pi$ be a space of smooth functions on $J(k) \backslash J(\mathbb{A})$ stable under the action of $J(\mathbb{A})$ by right translation, with $Z(\mathbb{A})$ acting by the character $\psi$. For every $\phi \in S(\mathbb{A})$ and $f \in \Pi$, the function $h \mapsto f(h g) \theta_{\bar{\psi}}^{\phi}(h g)$, where $h \in H(\mathbb{A})$, is left $Z(\mathbb{A})$-invariant, hence it can be viewed as a function on $V=H / Z$. Let $F J_{\psi}(\Pi)$ be the representation of $M(\mathbb{A})$ spanned by the functions

$$
F_{f, \phi}(g):=\int_{V(k) \backslash V(\mathbb{A})} f(v g) \theta_{\bar{\psi}}^{\phi}(v g) d v
$$


where $f$ runs over $\Pi$ and $\phi$ runs over $S(Y(\mathbb{A}))$. Using the definition of $\theta_{\bar{\psi}}^{\phi}$ and that $f$ is left $\xi$-invariant for all $\xi \in Y(k)$, the expression for $F_{f, \phi}(g)$ can be made more explicit as

$$
F_{f, \phi}(g)=\int_{X(k) \backslash V(\mathbb{A})} f(v g)\left[\rho_{\bar{\psi}}(v g) \phi\right](1) d v .
$$

The following is a global analogue of Proposition 4.1

Proposition 4.2. Let $k$ be a global field. Let $\Pi$ be a space of smooth functions on $J(k) \backslash J(\mathbb{A})$ stable under the action of $J(\mathbb{A})$ by right translation, with $Z(\mathbb{A})$ acting by the character $\psi$. If $F J_{\psi}(\Pi)$ is a Whittaker-generic representation of $M(\mathbb{A})$, then there exists $f \in \Pi$ and $u^{*}=\left(n^{*}, 0,1\right)$ where $n^{*} \in k^{\times}$such that

$$
\int_{U(k) \backslash U(\mathbb{A})} f(u) \bar{\psi}_{u *}(u) d u \neq 0 .
$$

Proof. For every $n^{*} \in k$ let $\psi_{n^{*}}$ be a character of $N(\mathbb{A})$ defined by $\psi_{n^{*}}(n)=\psi\left(n^{*} x\right)$ where $n=\left(\begin{array}{ll}1 & x \\ 0 & 1\end{array}\right)$. Since $F J_{\psi}(\Pi)$ is a Whittaker-generic representation of $M(\mathbb{A})$, there exist $f^{\prime} \in \Pi, \phi \in S(Y(\mathbb{A}))$ and $n^{*} \in k^{\times}$such that the following integral is non-vanishing:

$$
\int_{N(k) \backslash N(\mathbb{A})} \int_{X(k) \backslash V(\mathbb{A})} f^{\prime}(v n)\left[\rho_{\bar{\psi}}(v n) \phi\right](1) \bar{\psi}_{n *}(n) d v d n \neq 0 .
$$

Since $X(k) \backslash V(\mathbb{A})$ is a union of $X(k) \backslash X(\mathbb{A}) \cdot y$, where $y$ runs over $Y(\mathbb{A})$, there exists $y \in Y(\mathbb{A})$ such that

$$
\int_{N(k) \backslash N(\mathbb{A})} \int_{X(k) \backslash X(\mathbb{A})} f^{\prime}(x y n)\left[\rho_{\bar{\psi}}(x y n) \phi\right](1) \bar{\psi}_{n *}(n) d x d n \neq 0 .
$$

Since $y n=x^{\prime} n y$, for some element $x^{\prime} \in X(\mathbb{A})$, after changing the variable $x$, the previous expression is equivalent to

$$
\int_{N(k) \backslash N(\mathbb{A})} \int_{X(k) \backslash X(\mathbb{A})} f^{\prime}(x n y)\left[\rho_{\bar{\psi}}(x n y) \phi\right](1) \bar{\psi}_{n *}(n) d x d n \neq 0 .
$$

Since $\left[\rho_{\bar{\psi}}(x n y) \phi\right](1)=\phi(y)$, it follows that

$$
\int_{N(k) \backslash N(\mathbb{A})} \int_{X(k) \backslash X(\mathbb{A})} f^{\prime}(x n y) \bar{\psi}_{n *}(n) d x d n \neq 0 .
$$

Let $f$ be the right translate of $f^{\prime}$ by $y$. The conclusion of the proposition holds for this $f$.

Here is a simple criterion when the conditions of Propositions 4.1 and 4.2 are satisfied.

Lemma 4.3. If $k$ is a local field or a global field, assume that the action of $M$ on $\pi$ or the action of $M(\mathbb{A})$ on $\Pi$, respectively, descends to a d-fold central extension of $\mathrm{SL}_{2}(k)$ or $\mathrm{SL}_{2}(\mathbb{A})$, but not to a smaller degree cover. If $d$ is 1 and $m$ is odd, or $d$ is 2 and $m$ is even, or $d>2$ and $m$ is arbitrary, then the action of $M$ on $F J_{\psi}(\pi)$ or $M(\mathbb{A})$ on $F J_{\psi}(\Pi)$ does not descend to the linear quotient $\mathrm{SL}_{2}(k)$ or $\mathrm{SL}_{2}(\mathbb{A})$. In particular, it is a Whittaker-generic representation of $M$ or $M(\mathbb{A})$.

Proof. This is an easy consequence of the following well-known fact for local nonarchimedean fields. If $m$ is even, then $\rho_{\psi}$, as a representation of $M$, descends to the linear quotient $\mathrm{SL}_{2}(k)$. If $m$ is odd, then $\rho_{\psi}$ descends to a 2 -fold central extension of $\mathrm{SL}_{2}(k)$. 


\section{Nilpotent orbits}

Let $G$ be a central extension of degree $d$ of a reductive group over a local field $k$ of characteristic 0 or $p>0$. If the characteristic is $p$, we assume that $d$ is prime to $p$. Let $\mathfrak{g}$ be the Lie algebra of $G$. Let $\mathfrak{n}$ be a nilpotent subalgebra of $\mathfrak{g}$. If the characteristic of $k$ is 0 or $p$ is large enough, one can define a map exp $: \mathfrak{n} \rightarrow G$ whose image is a subgroup denoted by $N$. To that end, assume that $G$ is a linear group. Without loss of generality we can assume that $G$ is semi-simple. Then there exists a faithful, finite-dimensional representation $V$ of $G$ over $k$, and a tensor $T \in V^{\otimes^{n}} \otimes\left(V^{*}\right)^{\otimes^{m}}$ such that $G$ is defined as the set of all $g \in \operatorname{GL}(V)$ fixing $T$. Then $\mathfrak{g}$ is a subalgebra of $\operatorname{End}(V)$ and $x \cdot T=0$ for $x \in \mathfrak{g}$. If the characteristic of $k$ is 0 or $p$ is sufficiently large, then, for every $x \in \mathfrak{n}$, the usual exponential power series $\exp (x)$ is a well-defined finite $\operatorname{sum}$ and $\exp (x) \cdot T=T$. In particular, $\exp (x) \in G$. Let $N=\exp (\mathfrak{n})$. It is a subgroup of $G$ since, for $x, y \in \mathfrak{n}, \exp (x) \exp (y)=\exp (z)$ where $z \in \mathfrak{n}$ is given by the Campbell-Hausdorff formula

$$
z=x+y+\frac{1}{2}[x, y]+\frac{1}{12}[x,[x, y]]+\frac{1}{12}[y,[y, x]] \cdots
$$

which is a well-defined finite sum if $p$ is large enough. Any central extension of $G$ of degree $d$ prime to $p$ splits over $N$ and the splitting is unique since $N$ is a $d$-divisible group; see Appendix I in [MW95]. Hence we have defined the exponential map, provided that $p$ is large enough.

For example, if $V=\mathfrak{g}$, the Lie bracket is a map $\mathfrak{g} \otimes \mathfrak{g} \rightarrow \mathfrak{g}$, which is the same as an element in $\mathfrak{g} \otimes \mathfrak{g} \otimes \mathfrak{g}^{*}$, and by Proposition 5.5.5 in Ca93] exp : $\mathfrak{n} \rightarrow \operatorname{Aut}(\mathfrak{g})$ is well defined if $p>3(h-1)$ where $h$ is the Coxeter number. On the other hand, assume that the characteristic of $k$ is not 2 and $G$ is an orthogonal or symplectic group acting on a space $V$ with a non-degenerate orthogonal or symplectic bilinear form. Then $G$ is the group of all $g \in \mathrm{GL}(V)$ such that $i(g)=g^{-1}$ where $i$ is an involution of the associative algebra $\operatorname{End}(V)$, arising from the bilinear form. The Lie algebra consists of $x \in \operatorname{End}(V)$ such that $i(x)=-x$. In this case the map exp $: \mathfrak{n} \rightarrow G$ is well defined if $p \geq \operatorname{dim} V$.

Let $\psi$ be a smooth character of $k$ and $\kappa$ the Killing form on $\mathfrak{g}$. Let $u$ be a nilpotent element in $\mathfrak{g}$. Then $u$ defines a function

$$
\psi_{u}: \mathfrak{g} \rightarrow \mathbb{C}^{\times}
$$

by

$$
\psi_{u}(x)=\psi(\kappa(u, x)) .
$$

If $\kappa(u,[x, y])=0$ for all $x, y \in \mathfrak{n}$, then, by the Campbell-Hausdorff formula, $\exp (x) \mapsto \psi_{u}(x)$ defines a character of $N=\exp (\mathfrak{n})$. Abusing notation, we shall use $\psi_{u}$ to denote this character of $N$. One prominent example arises as follows. We assume that the theorem of Jacobson-Morozov holds, i.e., there exists a map $\varphi: \mathfrak{s l}_{2} \rightarrow \mathfrak{g}$ such that

$$
u=\varphi\left(\begin{array}{ll}
0 & 0 \\
1 & 0
\end{array}\right)
$$

Moreover, if $G$ is linear, we assume that $p$ is large enough so that the map $\varphi$ lifts to a map $\varphi: \mathrm{SL}_{2} \rightarrow G$. More precisely, if $(\bar{u}, s, u)$ is the $\mathfrak{s l}_{2}$-triple furnished by $\varphi$, we need that $\exp (t \bar{u})$ and $\exp (t u)$ are well defined and contained in $G$, for all $t \in k$, 
and then $\varphi: \mathrm{SL}_{2} \rightarrow G$ is defined by

$$
\varphi\left(\begin{array}{ll}
1 & 0 \\
t & 1
\end{array}\right)=\exp (t u) \text { and } \varphi\left(\begin{array}{ll}
1 & t \\
0 & 1
\end{array}\right)=\exp (t \bar{u})
$$

If we pass to a central extension of $G$, then $\varphi: \mathrm{SL}_{2} \rightarrow G$ lifts to a map from the universal central extension of $\mathrm{SL}_{2}$, denoted by $M$, to the central extension of $G$. The group of characters of the torus of diagonal matrices in $\mathrm{SL}_{2}$ is identified with $\mathbb{Z}$ where $j \in \mathbb{Z}$ defines a character

$$
\left(\begin{array}{cc}
t & 0 \\
0 & t^{-1}
\end{array}\right) \mapsto t^{j}
$$

for all $t \in k^{\times}$. The adjoint action of $\varphi\left(\mathrm{SL}_{2}\right)$ on $\mathfrak{g}$ gives a $\mathbb{Z}$-grading of $\mathfrak{g}$. More precisely, let $j \in \mathbb{Z}$; then $x \in \mathfrak{g}$ has a $\varphi$-weight $j$ if the $\varphi\left(\begin{array}{cc}t & 0 \\ 0 & t^{-1}\end{array}\right)$-conjugate of $x$ is equal to $t^{j} \cdot x$ for all $t \in k^{\times}$. Let $\mathfrak{g}(j)$ be the set of all $x$ of $\varphi$-weight $j$. Note that $u \in \mathfrak{g}(-2)$. Let

$$
\mathfrak{n}_{u}=\bigoplus_{j \geq 2} \mathfrak{g}(j)
$$

Since $u \in \mathfrak{g}(-2),\left[\mathfrak{n}_{u}, \mathfrak{n}_{u}\right] \subseteq \bigoplus_{j \geq 3} \mathfrak{g}(j)$ and two weight spaces are perpendicular with respect to $\kappa$, unless the weights are opposite; it follows that $\kappa(u,[x, y])=0$ for all $x, y \in \mathfrak{n}_{u}$. Hence $\psi_{u}$ defines a character of $N_{u}=\exp \left(\mathfrak{n}_{u}\right)$. The pair $\left(N_{u}, \psi_{u}\right)$ is precisely the one discussed in the introduction.

In a similar fashion, if $k$ is a global field and $\psi$ is a smooth character of $\mathbb{A}$ trivial on $k$, then any nilpotent element $u$ in $\mathfrak{g}$ defines a character $\psi_{u}$ of $N_{u}(\mathbb{A})$ trivial on $N_{u}(k)$.

\section{RAISING NILPOTENT ORBITS}

We continue with the set up of the previous section. Let $\mathfrak{c} \subseteq \mathfrak{g}$ be the centralizer of $\varphi\left(\mathfrak{s l}_{2}\right)$ in $\mathfrak{g}$. Assume we have a non-trivial map

$$
\varphi_{\mathfrak{c}}: \mathfrak{s l}_{2} \rightarrow \mathfrak{c} .
$$

Let $\mathfrak{s l}_{2, \mathfrak{c}}=\varphi_{\mathfrak{c}}\left(\mathfrak{s l}_{2}\right)$ and

$$
u_{\mathfrak{c}}=\varphi_{\mathfrak{c}}\left(\begin{array}{ll}
0 & 0 \\
\nu & 0
\end{array}\right) \in \mathfrak{s l}_{2, \mathfrak{c}}
$$

for some $\nu \neq 0$. The map $\varphi_{\mathfrak{c}}$ lifts to a map $\varphi_{\mathfrak{c}}: M \rightarrow G$ where $M$ is the universal central extension of $\mathrm{SL}_{2}$. Let

$$
\varphi^{\prime}=\varphi \oplus \varphi_{\mathfrak{c}}: \mathfrak{s l}_{2} \rightarrow \mathfrak{g} .
$$

This is a Jacobson-Morozov map corresponding to the nilpotent element $u^{\prime}=u+u_{\mathfrak{c}}$. Let $\mathfrak{g}(j, l)$ be the subspace of $\mathfrak{g}$ of all elements of $\varphi$-weight $j$ and $\varphi_{\mathfrak{c}}$-weight $l$. Note that $\mathfrak{n}_{u}$, defined in the previous section, is a sum of $\mathfrak{g}(j, l)$ such that $j \geq 2$, while $\mathfrak{n}_{u^{\prime}}$ is a sum of $\mathfrak{g}(j, l)$ such that $j+l \geq 2$.

Assume the following:

(1) The $\varphi_{\mathfrak{c}}$-weights $l$ are bounded by 2 .

(2) Under the action of $\mathfrak{s l}_{2, \mathfrak{c}}$

$$
\mathfrak{g}(1)=\mathfrak{g}(1)^{\mathfrak{s l}_{2, \mathfrak{c}}} \oplus m V_{2} .
$$

(3) $\operatorname{dim} \mathfrak{g}(0,2)=\operatorname{dim} \mathfrak{g}(2,2)+1$. 
Here $V_{2}$ denotes the irreducible 2-dimensional representation of $\mathfrak{s l}_{2}$. Let $\log X=$ $\mathfrak{g}(1,1)$ and $\log Y=\mathfrak{g}(1,-1)$. Let $\mathfrak{h}=\log X \oplus \log Y \oplus \mathfrak{n}_{u}$ and $H=\exp (\mathfrak{h})$. Let $N_{u}^{\prime}$ be the codimension-one subgroup of $N_{u}$ such that $\psi_{u}$ is trivial on $N_{u}^{\prime}$. Then $H / N_{u}^{\prime}$ is a Heisenberg group of dimension $2 m+1$ with the center $N_{u} / N_{u}^{\prime}$. Let $J$ be the semi-direct product of $H / N_{u}^{\prime}$ and $M$, where $M$ acts by conjugation on $H / N_{u}^{\prime}$ via the map $\varphi_{\mathfrak{c}}: M \rightarrow G$. It is a Jacobi group. Let $\pi$ be a smooth representation of $G$. Then $\pi_{N_{u}, \psi_{u}}$ is a representation of $J$. Assume that $\pi_{N_{u}, \psi_{u}} \neq 0$. If the natural action of $M$ on $F J_{\psi_{u}}\left(\pi_{N_{u}, \psi_{u}}\right)=\operatorname{Hom}_{H}\left(\rho_{\psi_{u}}, \pi_{N_{u}, \psi_{u}}\right)$ is Whittaker-generic, i.e., not a multiple of the trivial representation, then we can raise the orbit. More precisely, we have the following.

Proposition 6.1. Assume that $k$ is a non-archimedean local field and conditions (1)-(3) above are all satisfied. Let $\pi$ be a smooth representation of $G$ such that $\pi_{N_{u}, \psi_{u}} \neq 0$. Assume that $F J_{\psi_{u}}\left(\pi_{N_{u}, \psi_{u}}\right)$ is a Whittaker-generic representation of $M$. Then $\pi_{N_{u^{\prime}}, \psi_{u^{\prime}}} \neq 0$, where $u^{\prime}=u+u_{\mathfrak{c}}$, for some choice of nilpotent $u_{\mathfrak{c}} \neq 0$ in $\mathfrak{s l}_{2, \mathfrak{c}}$.

Proof. Let $\mathfrak{n}_{\mathfrak{c}} \subset \mathfrak{s l}_{2, \mathfrak{c}}$ be the subspace of elements of $\varphi_{\mathfrak{c}}$-weight 2 and let $N_{\mathfrak{c}}=$ $\exp \left(\mathfrak{n}_{\mathfrak{c}}\right) \subset M$. Let $\mathfrak{u}=\mathfrak{n}_{\mathfrak{c}} \oplus \log X \oplus \mathfrak{n}_{u}$ and $U=\exp (\mathfrak{u})$.

Since $F J_{\psi_{u}}\left(\pi_{N_{u}, \psi_{u}}\right)$ is a Whittaker-generic representation of $M$, by Proposition 4.1. $\pi_{U, \tilde{\psi}} \neq 0$ for a character $\tilde{\psi}$ of $U$, equal to $\psi_{u}$ on $N_{u}$, trivial on $X$ and equal to $\psi_{u_{\mathfrak{c}}}$ on $N_{\mathfrak{c}}$ for some choice of nilpotent $u_{\mathfrak{c}} \neq 0$ in $\mathfrak{s l}_{2, \mathfrak{c}}$. In the remainder of the proof we shall "transfer" the character $\tilde{\psi}$ from $U$ to $N_{u^{\prime}}$ using a Heisenberg group.

Consider the sum $\bigoplus_{j} \mathfrak{g}(j, 2)$ over all $j$. It is a $\varphi\left(\mathfrak{s l}_{2}\right)$-module. By representation theory of $\mathfrak{s l}_{2}$, the map $x \rightarrow[u, x]$ is an injection of $\mathfrak{g}(2,2)$ into $\mathfrak{g}(0,2)$ and the complement of the image is spanned by $\varphi\left(\mathfrak{s l}_{2}\right)$-fixed vectors. Since $\mathfrak{n}_{\mathfrak{c}}$ is fixed by $\varphi\left(\mathfrak{s l}_{2}\right)$ and $\operatorname{dim} \mathfrak{g}(0,2)=\operatorname{dim} \mathfrak{g}(2,2)+1$, by condition $(3)$, it follows that

$$
\mathfrak{g}(0,2)=\mathfrak{n}_{\mathfrak{c}} \oplus[u, \mathfrak{g}(2,2)] .
$$

Let $\mathfrak{u}^{\prime}$ be the space obtained from $\mathfrak{u}$ by removing the summand $\mathfrak{g}(2,-2)$ and adding $[u, \mathfrak{g}(2,2)]$, that is, $\mathfrak{u}^{\prime}$ is a direct sum of $\mathfrak{g}(0,2), \mathfrak{g}(1,1)$ and $\mathfrak{g}(j, l)$ for all $j \geq 2$ but not $(j, l)=(2,-2)$. Since $|l| \leq 2$, by condition $(1)$, it follows that $\mathfrak{u}^{\prime}$ is a subalgebra containing $\mathfrak{n}_{u^{\prime}} .\left(\mathfrak{n}_{u^{\prime}}\right.$ is a sum of $\mathfrak{g}(j, l)$ with $j+l \geq 2$.)

Lemma 6.2. Let $U^{\prime}=\exp \left(\mathfrak{u}^{\prime}\right)$. Then $\psi_{u^{\prime}}$ is a character of $U^{\prime}$ equal to $\tilde{\psi}$ on $U^{\prime} \cap U$.

Proof. To prove that $\psi_{u^{\prime}}$ is a character, we need to check $\kappa\left(u^{\prime},[x, y]\right)=0$ for all $x, y \in \mathfrak{u}^{\prime}$. Clearly, it suffices to prove vanishing when $x \in \mathfrak{g}(j, l)$ and $y \in \mathfrak{g}\left(j^{\prime}, l^{\prime}\right)$. Recall that $u \in \mathfrak{g}(-2,0)$ and $u_{\mathfrak{c}} \in \mathfrak{g}(0,-2)$. If $\kappa\left(u_{\mathfrak{c}},[x, y]\right) \neq 0$, then $j+j^{\prime}=0$. Hence $j=j^{\prime}=0$ and $x, y \in \mathfrak{g}(0,2)$. Then $[x, y]=0$, hence $\kappa\left(u_{\mathfrak{c}},[x, y]\right)=0$, a contradiction. If $\kappa(u,[x, y]) \neq 0$, then $j+j^{\prime}=2$ and $l+l^{\prime}=0$. But there is no such pair $\mathfrak{g}(j, l)$ and $\mathfrak{g}\left(j^{\prime}, l^{\prime}\right)$ in $\mathfrak{u}^{\prime}$. Hence $\psi_{u^{\prime}}$ is a character of $U^{\prime}$.

Note that $U^{\prime} \cap U=N_{\mathfrak{c}} X\left(N_{u} \cap U^{\prime}\right)$. Since both $\psi_{u^{\prime}}$ and $\tilde{\psi}$ are equal to $\psi_{u_{\mathrm{c}}}, 1$ and $\psi_{u}$ on the three factors, respectively, it follows that $\psi_{u^{\prime}}=\tilde{\psi}$ on $U^{\prime} \cap U$, as desired.

Let $\mathfrak{z}^{\prime}=\mathfrak{u} \cap \mathfrak{u}^{\prime}$ and let

$$
\mathfrak{h}^{\prime}=\mathfrak{u}^{\prime}+\mathfrak{u}=[u, \mathfrak{g}(2,2)] \oplus \mathfrak{g}(2,-2) \oplus \mathfrak{z}^{\prime} .
$$

Lemma 6.3. The pairing $\kappa\left(u^{\prime},[x, y]\right)$ where $x \in[u, \mathfrak{g}(2,2)]$ and $y \in \mathfrak{g}(2,-2)$ is non-degenerate. 
Proof. Since $[x, y] \in \mathfrak{g}(2)$ and $u_{\mathfrak{c}} \in \mathfrak{g}(0), \kappa\left(u^{\prime},[x, y]\right)=\kappa(u,[x, y])$. By the invariance of the Killing form, we have

$$
\kappa(u,[x, y])=\kappa(y,[u, x]) .
$$

The pairing is non-degenerate since $[u,[u, \mathfrak{g}(2,2)]]=\mathfrak{g}(-2,2)$, and the Killing form gives a non-degenerate pairing between $\mathfrak{g}(2,-2)$ and $\mathfrak{g}(-2,2)$.

Let $H^{\prime}=\exp \left(\mathfrak{h}^{\prime}\right)$ and $Z^{\prime}=\exp \left(\mathfrak{z}^{\prime}\right)$, i.e., $Z^{\prime}=U \cap U^{\prime}$. Let $Z^{\prime \prime}$ be the codimensionone subgroup of $Z^{\prime}$ where the character $\psi_{u^{\prime}}=\tilde{\psi}$ is trivial. Lemma 6.3 implies that $H^{\prime} / Z^{\prime \prime}$ is a Heisenberg group. Since $\pi_{U, \tilde{\psi}} \neq 0$, it follows that

$$
\pi_{Z^{\prime}, \tilde{\psi}}=\pi_{Z^{\prime}, \psi_{u^{\prime}}} \neq 0
$$

and $\pi_{U^{\prime}, \psi_{u^{\prime}}} \neq 0$ by Proposition [2.1] applied to $X=\exp ([u, \mathfrak{g}(2,2)])$ and $Y=$ $\exp (\mathfrak{g}(2,-2))$. Since $N_{u^{\prime}} \subseteq U^{\prime}$, the proposition follows.

Corollary 6.4. Assume that $k$ is a non-archimedean local field and conditions (1)-(3) above are all satisfied. Let $\pi$ be a smooth representation of $G$ such that $\pi_{N_{u}, \psi_{u}} \neq 0$. If the conditions of Lemma 4.3 are satisfied for the $M$-module $\pi_{N_{u}, \psi_{u}}$, then $\pi_{N_{u^{\prime}}, \psi_{u^{\prime}}} \neq 0$, where $u^{\prime}=u+u_{\mathfrak{c}}$, for some choice of nilpotent $u_{\mathfrak{c}} \neq 0$ in $\mathfrak{s l}_{2, \mathfrak{c}}$.

Remark. If $\pi$ is a representation of a linear group, the corollary simply states that we can raise the orbit if $m$, in condition (2), is odd.

Now assume that $k$ is a global field. Let $\Pi$ be a space of smooth functions on $G(k) \backslash G(\mathbb{A})$ stable under the action of $G(\mathbb{A})$ by right translation. Let $\Pi_{N_{u}, \psi_{u}}$ be the space of smooth functions on $J(k) \backslash J(\mathbb{A})$ consisting of

$$
\tilde{f}(g)=\int_{N_{u}(k) \backslash N_{u}(\mathbb{A})} f(n g) \bar{\psi}_{u}(n) d n
$$

where $f$ runs through $\Pi$. Similarly one can define $\Pi_{U, \tilde{\psi}}$, where $\tilde{\psi}$ is a character of $U$. Recall from Section 4 that one may then define $F J_{\psi_{u}}\left(\Pi_{N_{u}, \psi_{u}}\right)$, which is the representation of $M(\mathbb{A})$.

The following global version of Proposition 6.1 is proved using Propositions 2.2 and 4.2 (instead of Propositions 2.1 and 4.1). We omit the details.

Proposition 6.5. Assume that $k$ is a global field and conditions (1)-(3) above are all satisfied. Let $\Pi$ be a space of smooth functions on $G(k) \backslash G(\mathbb{A})$ that is stable under the action of $G(\mathbb{A})$ by right translation and has the property that $\Pi_{N_{u}, \psi_{u}} \neq 0$. Assume that $F J_{\psi_{u}}\left(\Pi_{N_{u}, \psi_{u}}\right)$ is a Whittaker-generic representation of $M(\mathbb{A})$. Then $\Pi_{N_{u^{\prime}}, \psi_{u^{\prime}}} \neq 0$, where $u^{\prime}=u+u_{\mathfrak{c}}$, for some choice of nilpotent $u_{\mathfrak{c}} \neq 0$ in $\mathfrak{s l}_{2, \mathfrak{c}}$.

We also have the following global analogue of Corollary 6.4 with a similar argument (using Proposition 6.5] instead of Proposition 6.1).

Corollary 6.6. Assume that $k$ is a global field and conditions (1)-(3) above are all satisfied. Let $\Pi$ be a space of smooth functions on $G(k) \backslash G(\mathbb{A})$ that is stable under the action of $G(\mathbb{A})$ by right translation and has the property that $\Pi_{N_{u}, \psi_{u}} \neq 0$. If the conditions of Lemma 4.3 are satisfied for the $M(\mathbb{A})$-module $\Pi_{N_{u}, \psi_{u}} \neq 0$, then $\Pi_{N_{u^{\prime}}, \psi_{u^{\prime}}} \neq 0$, where $u^{\prime}=u+u_{\mathfrak{c}}$, for some choice of nilpotent $u_{\mathfrak{c}} \neq 0$ in $\mathfrak{s l}_{2, \mathfrak{c}}$. 


\section{Symplectic-orthogonal groups}

Let $W$ be a vector space over a field $k$ (of sufficiently large characteristic) equipped with a non-degenerate symmetric or skew-symmetric bilinear form $\langle$,$\rangle .$ Let $\mathfrak{g}$ be the Lie algebra of endomorphisms of $W$ preserving the bilinear form. Let

$$
i: W \otimes W \rightarrow \operatorname{End}(W)
$$

be a map defined by

$$
i(x \otimes y)(z)=\langle y, z\rangle x-\langle z, x\rangle y
$$

for all $x, y, z \in W$. If $\langle$,$\rangle is symmetric, then i$ gives a bijection between $\bigwedge^{2} W$, the exterior square of $W$, and $\mathfrak{g}$. If $\langle$,$\rangle is skew-symmetric, then i$ gives a bijection between $S^{2}(W)$, the symmetric square of $W$, and $\mathfrak{g}$.

Let $V_{j}$ be the irreducible representation of $\mathfrak{s l}_{2}$ of dimension $j$. Then $V_{j}$ admits a unique, up to a non-zero scalar, $\mathfrak{s l}_{2}$-invariant bilinear form $\langle,\rangle_{j}$. This form is symmetric if $j$ is odd, and skew-symmetric if $j$ is even. We normalize $\langle,\rangle_{j}$ as follows. Let $v_{j} \in V_{j}$ be a non-zero highest weight vector. Let $u=\left(\begin{array}{ll}0 & 0 \\ 1 & 0\end{array}\right)$. Then $u^{j-1} v_{j}$ is a lowest weight vector. Hence the number

$$
a=\left\langle v_{j}, u^{j-1} v_{j}\right\rangle_{j}
$$

is non-zero. This number depends on the choice of $v_{j}$, however, its square class does not. In order to distinguish the forms, we shall write $\langle,\rangle_{j}^{a}$ for this form or simply $\langle,\rangle_{j}$ if $a$ is in the class of 1 . This normalization differs from the one in Wal01 by the factor $(-1)^{[(i-1) / 2]}$.

Let $\varphi: \mathfrak{s l}_{2} \rightarrow \mathfrak{g}$. Then, under the action of $\varphi\left(\mathfrak{s l}_{2}\right)$, the symplectic space $W$ can be decomposed as

$$
\begin{aligned}
W & =\bigoplus_{j} V_{j} \otimes U_{j}, \\
\langle,\rangle & =\bigoplus_{j}\langle,\rangle_{j} \otimes(,)_{j}
\end{aligned}
$$

where

$$
U_{j}=\operatorname{Hom}_{\mathfrak{s l}_{2}}\left(V_{j}, W\right)
$$

with the form $(,)_{j}$. The nilpotent orbit of $u$ gives a partition $\underline{p}$ of $\operatorname{dim}(W)$ in which $j$ appears with multiplicity $\operatorname{dim} U_{j}$. If the form on $W$ is symmetric, then the forms $(,)_{j}$ and $\langle,\rangle_{j}$ have the same signs. Otherwise the two forms have different signs. If $(,)_{j}$ is skew-symmetric, then $\operatorname{dim}\left(U_{j}\right)$ is even. Thus $j$ in $p$ must have even multiplicity. The conjugacy class of $u$ is determined by the partition $\underline{p}$ and isomorphism classes of $\left(U_{j},(,)_{j}\right)$.

Put $W_{j}=U_{j} \otimes V_{j}$ in the decomposition (7.1). If $\langle$,$\rangle is symmetric, then$

$$
\begin{aligned}
& \mathfrak{g} \cong \bigwedge^{2}(W) \cong\left(\bigoplus_{j} \bigwedge^{2}\left(W_{j}\right)\right) \oplus\left(\bigoplus_{i<j} W_{i} \otimes W_{j}\right), \\
& \bigwedge^{2}\left(W_{j}\right) \cong \bigwedge^{2}\left(V_{j}\right) \otimes S^{2}\left(U_{j}\right) \oplus S^{2}\left(V_{j}\right) \otimes \bigwedge^{2}\left(U_{j}\right) .
\end{aligned}
$$

If $\langle$,$\rangle is skew-symmetric, then$

$$
\begin{aligned}
& \mathfrak{g} \cong S^{2}(W) \cong\left(\bigoplus_{i} S^{2}\left(W_{j}\right)\right) \oplus\left(\bigoplus_{i<j} W_{i} \otimes W_{j}\right), \\
& S^{2}\left(W_{j}\right) \cong S^{2}\left(V_{j}\right) \otimes S^{2}\left(U_{j}\right) \oplus \bigwedge^{2}\left(V_{j}\right) \otimes \bigwedge^{2}\left(U_{j}\right) .
\end{aligned}
$$

If the decomposition (7.1) of $W$ contains a non-trivial summand such that the form $(,)_{i}$ is skew-symmetric, then $\operatorname{Sp}\left(U_{i}\right)$ acts naturally on $W$ preserving the form $\langle$,$\rangle . This gives an embedding of \operatorname{Sp}\left(U_{i}\right)$ into $G$. The adjoint action of $\operatorname{Sp}\left(U_{i}\right)$ on $\mathfrak{g}$ commutes with $\varphi\left(\mathfrak{s l}_{2}\right)$. In particular, $\operatorname{Sp}\left(U_{i}\right)$ acts on each $\varphi$-weight space $\mathfrak{g}(j)$. 
Lemma 7.1. Assume that the decomposition (7.1) of $W$ contains a non-trivial summand such that the form $(,)_{i}$ is skew-symmetric. Let $\varphi_{\mathfrak{c}}: \mathrm{SL}_{2} \rightarrow \operatorname{Sp}\left(U_{i}\right)$ be a map corresponding to a long root of $\operatorname{Sp}\left(U_{i}\right)$. Let $\mathrm{SL}_{2, \mathfrak{c}}$ be the image of $\varphi_{\mathfrak{c}}$. Then, under the adjoint action of $\mathrm{SL}_{2, \mathfrak{c}}, \mathfrak{g}(1)$ decomposes as

$$
\mathfrak{g}(1) \cong \mathfrak{g}(1)^{\mathrm{SL}_{2, \mathfrak{c}}} \oplus m V_{2}
$$

where, if $i$ is odd,

$$
m=i\left(\sum_{j>i, j \text { even }} \operatorname{dim} U_{j}\right) \oplus\left(\sum_{j<i, j \text { even }} j \operatorname{dim} U_{j}\right)
$$

and, if $i$ is even,

$$
m=i\left(\sum_{j>i, j \text { odd }} \operatorname{dim} U_{j}\right) \oplus\left(\sum_{j<i, j \text { odd }} j \operatorname{dim} U_{j}\right)
$$

Proof. Under the action of $\mathrm{SL}_{2, \mathfrak{c}}$, the space $U_{i}$ decomposes as $V_{2} \oplus U_{i}^{\mathrm{SL}_{2, \mathfrak{c}}}$ where $U_{i}^{\prime}=U_{i}^{\mathrm{SL}_{2, \mathrm{c}}}$ is a non-degenerate symplectic space. It follows from (7.2) and (7.3) that

$$
\bigoplus_{j \neq i}\left(\left(V_{i} \otimes V_{j}\right)(1)\right) \otimes\left(V_{2} \otimes U_{j}\right)
$$

is the complement of $\mathfrak{g}(1)^{\mathrm{SL}_{2, \mathfrak{c}}}$ in $\mathfrak{g}(1)$. On the other hand, $\left(V_{i} \otimes V_{j}\right)(1) \neq 0$ only for $i$ and $j$ of different parity, and then

$$
\operatorname{dim}\left(\left(V_{i} \otimes V_{j}\right)(1)\right)=\min \{i, j\} .
$$

The lemma follows.

If the decomposition (7.1) of $W$ contains a non-trivial summand such that the form $(,)_{i}$ is symmetric, then $\mathrm{O}\left(U_{i}\right)$ acts naturally on $W$ preserving the form $\langle$,$\rangle .$ This gives an embedding of $\mathrm{O}\left(U_{i}\right)$ into $G$. The adjoint action of $\mathrm{O}\left(U_{i}\right)$ on $\mathfrak{g}$ commutes with $\varphi\left(\mathfrak{s l}_{2}\right)$. In particular, $\mathrm{O}\left(U_{i}\right)$ acts on each $\varphi$-weight space $\mathfrak{g}(j)$.

Lemma 7.2. Assume that the decomposition (7.1) of $W$ contains a non-trivial summand for $i$ such that the form $(,)_{i}$ is symmetric with dimension $\geq 4$, and has a 2-dimensional isotropic subspace. Then $\mathrm{O}\left(U_{i}\right)$ has a parabolic subgroup fixing this subspace with Levi subgroup isomorphic to $\mathrm{GL}_{2} \times \mathrm{O}\left(U_{i}^{\prime}\right)$, where $U_{i}^{\prime}=U_{i}^{\mathrm{GL}_{2}}$. Let $\varphi_{\mathfrak{c}}: \mathrm{SL}_{2} \rightarrow \mathrm{O}\left(U_{i}\right)$ be a map corresponding to the roots of $\mathrm{GL}_{2}$. Let $\mathrm{SL}_{2, \mathfrak{c}}$ be the image of $\varphi_{\mathfrak{c}}$. Then the structure of the $\mathrm{SL}_{2, \mathfrak{c}}$-module $\mathfrak{g}(1)$ is given by

$$
\mathfrak{g}(1) \cong \mathfrak{g}(1)^{\mathrm{SL}_{2, \mathfrak{c}}} \oplus 2 m V_{2}
$$

where, if $i$ is odd,

$$
m=i\left(\sum_{j>i, j \text { even }} \operatorname{dim} U_{j}\right) \oplus\left(\sum_{j<i, j \text { even }} j \operatorname{dim} U_{j}\right)
$$

and, if $i$ is even,

$$
m=i\left(\sum_{j>i, j \text { odd }} \operatorname{dim} U_{j}\right) \oplus\left(\sum_{j<i, j \text { odd }} j \operatorname{dim} U_{j}\right)
$$

Proof. Under the action of $\mathrm{SL}_{2, \mathfrak{c}}$, the space $U_{i}$ decomposes as $V_{2} \otimes U_{2} \oplus U_{i}^{\mathrm{SL}_{2, \mathfrak{c}}}$ where $U_{i}^{\mathrm{SL}_{2, \mathrm{c}}}=U_{i}^{\prime}$ and $U_{2}$ is a 2-dimensional symplectic space. It follows from (17.2) and (7.3) that

$$
\bigoplus_{j \neq i}\left(\left(V_{i} \otimes V_{j}\right)(1)\right) \otimes\left(\left(V_{2} \otimes U_{2}\right) \otimes U_{j}\right)
$$


is the complement of $\mathfrak{g}(1)^{\mathrm{SL}_{2, \mathfrak{c}}}$ in $\mathfrak{g}(1)$. On the other hand, $\left(V_{i} \otimes V_{j}\right)(1) \neq 0$ only for $i$ and $j$ of different parity, and then

$$
\operatorname{dim}\left(\left(V_{i} \otimes V_{j}\right)(1)\right)=\min \{i, j\} .
$$

The lemma follows.

\section{RAISING NILPOTENT ORBITS FROM SYMPLECTIC STABILIZERS}

Let $G=\operatorname{Sp}(W)$ or $\widetilde{\mathrm{Sp}}(W)$ if $W$ is a symplectic space, or $G=\mathrm{O}(W)$ if $W$ is an orthogonal space. Assume that we are in the situation as in Lemma 7.1, that is, the decomposition (7.1) of $W$ contains a non-trivial summand for $i$ such that the form $(,)_{i}$ is skew-symmetric. Let $\varphi_{\mathfrak{c}}: \mathfrak{s l}_{2} \rightarrow \mathfrak{s p}\left(U_{i}\right)$ be a map corresponding to a long root of $\mathfrak{s p}\left(U_{i}\right)$. Let

$$
\varphi^{\prime}=\varphi \oplus \varphi_{\mathfrak{c}}: \mathfrak{s l}_{2} \rightarrow \mathfrak{g}
$$

and let $u^{\prime}=u+u_{\mathfrak{c}}$ be a nilpotent element corresponding to $\varphi^{\prime}$. Since the orbit of $u_{\mathfrak{c}}$ in $\mathfrak{s l}_{2, \mathfrak{c}}=\varphi_{\mathfrak{c}}\left(\mathfrak{s l}_{2}\right)$ contains 0 in its closure, it follows that the closure of the orbit of $u^{\prime}$ contains the orbit of $u$.

We shall now determine the partition $p^{\prime}$ corresponding to $u^{\prime}$. From the decomposition (7.1) of $W$ under the action of $\varphi\left(\mathfrak{s l}_{2}\right)$ one can easily obtain a similar decomposition for $\varphi^{\prime}\left(\mathfrak{s l}_{2}\right)$. Indeed, under the action of $\mathfrak{s l}_{2, \mathfrak{c}}$, the symplectic space $U_{i}$ decomposes $U_{i}=V_{2} \oplus U_{i}^{\mathfrak{s l}_{2, \mathfrak{c}}}$ where $V_{2}$ is the irreducible 2-dimensional representation of $\mathfrak{s l}_{2}$. Now the Clebsch-Gordan formula $V_{i} \otimes V_{2} \cong V_{i-1} \oplus V_{i+1}$ implies that the partition $p^{\prime}$ is obtained from the partition $p$, corresponding to $u$, by replacing a pair $(i, i)$ by $(i+1, i-1)$.

More refined information about the conjugacy class of $u^{\prime}$ is given by the following proposition.

Proposition 8.1. Let $U_{j}$ be the spaces in the decomposition of $W$ under the action of $\varphi\left(\mathfrak{s l}_{2}\right)$. Then the spaces $U_{j}^{\prime}$ in the decomposition of $W$ under the action of $\varphi^{\prime}\left(\mathfrak{s l}_{2}\right)$ are

$$
U_{i}^{\prime}=U_{i}^{\mathfrak{s l} \mathfrak{l}_{2, \mathfrak{c}}}, U_{i+1}^{\prime}=U_{i+1} \oplus\langle b\rangle, U_{i-1}^{\prime}=U_{i-1} \oplus\langle b\rangle,
$$

for some $b \in k^{\times}$, and $U_{j}^{\prime}=U_{j}$ otherwise. Here $\langle b\rangle$ is the 1-dimensional orthogonal space with the form $b x^{2}$.

Proof. Let $v_{2} \in V_{2} \subseteq U_{i}$ be a highest weight vector. Let $a=\left(v_{2}, u_{\mathfrak{c}} v_{2}\right)_{i}$, so that the restriction of $(,)_{i}$ to $V_{2}$ is the form previously denoted as $\langle,\rangle_{2}^{a}$. The proposition follows from the following lemma, with $b=a i$.

Lemma 8.2. With respect to the decomposition $V_{i} \otimes V_{2} \cong V_{i-1} \oplus V_{i+1}$, the form $\langle,\rangle_{i} \otimes\langle,\rangle_{2}^{a}$ decomposes as

$$
\langle,\rangle_{i} \otimes\langle,\rangle_{2}^{a}=\langle,\rangle_{i+1}^{a i} \oplus\langle,\rangle_{i-1}^{a i}
$$

Proof. Let $v_{i} \in V_{i}$ be a highest weight vector such that $\left\langle v_{i}, u^{i-1} v_{i}\right\rangle=1$. Then

$$
v_{i+1}=v_{i} \otimes v_{2}
$$

is a highest weight vector of $V_{i+1}$. Using the Newton-Leibniz rule,

$$
\left(u+u_{\mathfrak{c}}\right)^{i} v_{i+1}=i \cdot\left(u^{i-1} v_{i} \otimes u_{\mathfrak{c}} v_{2}\right) .
$$

It follows that the pairing $\langle,\rangle_{i} \otimes\langle,\rangle_{2}^{a}$ evaluated at $v_{i+1}$ and $\left(u+u_{\mathfrak{c}}\right)^{i} v_{i+1}$ gives $a i$. Similarly,

$$
v_{i-1}=(i-1) \cdot\left(v_{i} \otimes u_{\mathfrak{c}} v_{2}\right)-u v_{i} \otimes v_{2}
$$


is a highest weight vector of $V_{i-1}$. Then

$$
\left(u+u_{\mathfrak{c}}\right)^{i-2} v_{i-1}=u^{i-2} v_{i} \otimes u_{\mathfrak{c}} v_{2}-u^{i-1} v_{i} \otimes v_{2} .
$$

Next, using $\left\langle-u v_{i}, u^{i-2} v_{i}\right\rangle_{i}=\left\langle v_{i}, u^{i-1} v_{i}\right\rangle_{i}=1$, the pairing $\langle,\rangle_{i} \otimes\langle,\rangle_{2}^{a}$ evaluated at $v_{i-1}$ and $\left(u+u_{\mathfrak{c}}\right)^{i-2} v_{i-1}$ also gives $a i$.

We shall now describe $\mathfrak{g}(j, l)$. Using the decompositions (7.2), (7.3) and $U_{i}=$ $V_{2} \oplus U_{i}^{\mathfrak{s l}_{2, \mathfrak{c}}}$ we see that $\mathfrak{g}(j, l) \neq 0$ only for $|l| \leq 2$. In particular, $\mathfrak{g}(1,1)$ and $\mathfrak{g}(0,2)$ are the only two such spaces contained in $\mathfrak{n}_{u^{\prime}}$ but not in $\mathfrak{n}_{u}$. Let

$$
E_{i}=\left\{\begin{array}{l}
\bigwedge^{2} V_{i} \text { if } i \text { is even, } \\
S^{2} V_{i} \text { if } i \text { is odd }
\end{array}\right.
$$

Then

$$
\mathfrak{g}(j, \pm 2) \cong E_{i}(j) \otimes S^{2}\left(V_{2}\right)( \pm 2)
$$

From (8.1), one can easily see that $\operatorname{dim} \mathfrak{g}(0,2)=\operatorname{dim} \mathfrak{g}(2,2)+1$, that is, condition (3) in Section 6 is satisfied. Therefore, by Lemma 7.1] all conditions (1)-(3) in Section 6 are satisfied. The following proposition follows from Proposition 6.1.

Proposition 8.3. Assume that we are in the situation as in Lemma 7.1 and $k$ is a non-archimedean local field. Let $\pi$ be a smooth representation of $G$. If $G=$ $\widetilde{\operatorname{Sp}}(W)$ assume that $\pi$ is a genuine representation. Let $u$ be a nilpotent element in $\mathfrak{g}$ such that $\pi_{N_{u}, \psi_{u}} \neq 0$. Assume that $F J_{\psi_{u}}\left(\pi_{N_{u}, \psi_{u}}\right)=\operatorname{Hom}_{H_{u, c}}\left(\rho_{\psi_{u}}, \pi_{N_{u}, \psi_{u}}\right)$ is a Whittaker-generic representation of $M$. Then $\pi_{N_{u^{\prime}}, \psi_{u^{\prime}}} \neq 0$ for a nilpotent element $u^{\prime}$ whose partition is obtained from the partition corresponding to $u$ via replacing a pair $(i, i)$ by $(i+1, i-1)$. 6.5 .

There is also a global version of Proposition 8.3. which follows from Proposition

Proposition 8.4. Assume that we are in the situation as in Lemma 7.1 and $k$ is a global field. Let $\Pi$ be a space of smooth functions on $G(k) \backslash G(\mathbb{A})$ stable under the action of $G(\mathbb{A})$ by right translation. If $G=\widetilde{\mathrm{Sp}}(W)$ assume that $\Pi$ consists of genuine functions. Let $u$ be a nilpotent element in $\mathfrak{g}$ such that $\Pi_{N_{u}, \psi_{u}} \neq 0$. Assume that F $J_{\psi_{u}}\left(\Pi_{N_{u}, \psi_{u}}\right)$ (see Section 5 for the definition) is a Whittaker-generic representation of $M(\mathbb{A})$. Then $\Pi_{N_{u^{\prime}}, \psi_{u^{\prime}}} \neq 0$ for a nilpotent element $u^{\prime}$ whose partition is obtained from the partition corresponding to $u$ via replacing a pair $(i, i)$ by $(i+1, i-1)$.

\section{RAISING NILPOTENT ORBITS FROM ORTHOGONAL STABILIZERS}

Let $G=\operatorname{Sp}(W)$ or $\widetilde{\mathrm{Sp}}(W)$ if $W$ is a symplectic space, or $G=\mathrm{O}(W)$ if $W$ is an orthogonal space. Assume that we are in the situation as in Lemma 7.2, that is, the decomposition (7.1) of $W$ contains a non-trivial summand for $i$ such that the form $(,)_{i}$ is symmetric with dimension $\geq 4$, and has a 2 -dimensional isotropic subspace. Then $\mathrm{O}\left(U_{i}\right)$ has a parabolic subgroup fixing this subspace with Levi subgroup isomorphic to $\mathrm{GL}_{2} \times \mathrm{O}\left(U_{i}^{\prime}\right)$ where $U_{i}^{\prime}=U_{i}^{\mathrm{GL}_{2}}$. Let $\varphi_{\mathfrak{c}}: \mathrm{SL}_{2} \rightarrow \mathrm{O}\left(U_{i}\right)$ be a map corresponding to the roots of $\mathrm{GL}_{2}$. Let

$$
\varphi^{\prime}=\varphi \oplus \varphi_{\mathfrak{c}}: \mathfrak{s l}_{2} \rightarrow \mathfrak{g}
$$

and let $u^{\prime}=u+u_{\mathfrak{c}}$ be a nilpotent element corresponding to $\varphi^{\prime}$. Since the orbit of $u_{\mathfrak{c}}$ in $\mathfrak{s l}_{2, \mathfrak{c}}=\varphi_{\mathfrak{c}}\left(\mathfrak{s l}_{2}\right)$ contains 0 in its closure, it follows that the closure of the orbit 
of $u^{\prime}$ contains the orbit of $u$. Since $U_{i}=2 V_{2} \oplus U_{i}^{\mathfrak{s l}_{2, \mathfrak{c}}}$, the Clebsch-Gordan formula implies that the partition $p^{\prime}$ corresponding to $u^{\prime}$ is obtained from the partition $p$, corresponding to $u$, by replacing a quadruple $(i, i, i, i)$ by $(i+1, i+1, i-1, i-1)$.

Next, let

$$
\begin{aligned}
& E_{i}=\left\{\begin{array}{l}
\bigwedge^{2} V_{i} \text { if } i \text { is even, } \\
S^{2} V_{i} \text { if } i \text { is odd },
\end{array}\right. \\
& F_{i}=\left\{\begin{array}{l}
S^{2} V_{i} \text { if } i \text { is even, } \\
\bigwedge^{2} V_{i} \text { if } i \text { is odd }
\end{array}\right.
\end{aligned}
$$

Then

$$
\mathfrak{g}(j, \pm 2) \cong E_{i}(j) \otimes \bigwedge^{2}\left(2 V_{2}\right)( \pm 2) \oplus F_{i}(j) \otimes S^{2}\left(2 V_{2}\right)( \pm 2)
$$

From (9.1), one can easily see that $\operatorname{dim} \mathfrak{g}(0,2)=\operatorname{dim} \mathfrak{g}(2,2)+1$, that is, condition (3) in Section 6 is satisfied. Therefore, by Lemma 7.2, all conditions (1)-(3) in Section 6 are satisfied. The following proposition follows directly from Proposition 6.1 and the discussion at the beginning of this section.

Proposition 9.1. Assume that we are in the situation as in Lemma 7.2 and $k$ is a non-archimedean local field. Let $\pi$ be a smooth representation of $G$. If $G=$ $\widetilde{\mathrm{Sp}}(W)$ assume that $\pi$ is a genuine representation. Let $u$ be a nilpotent element in $\mathfrak{g}$ such that $\pi_{N_{u}, \psi_{u}} \neq 0$. Assume that $F J_{\psi_{u}}\left(\pi_{N_{u}, \psi_{u}}\right)=\operatorname{Hom}_{H_{u, c}}\left(\rho_{\psi_{u}}, \pi_{N_{u}, \psi_{u}}\right)$ is a Whittaker-generic representation of $M$. Then $\pi_{N_{u^{\prime}}, \psi_{u^{\prime}}} \neq 0$ for a nilpotent element $u^{\prime}$ whose partition is obtained from the partition corresponding to $u$ via replacing a quadruple $(i, i, i, i)$ by $(i+1, i+1, i-1, i-1)$.

There is also a global version of Proposition 9.1, which follows directly from Proposition 6.5 and the discussion at the beginning of this section.

Proposition 9.2. Assume that we are in the situation as in Lemma 7.2 and $k$ is a global field. Let $\Pi$ be a space of smooth functions on $G(k) \backslash G(\mathbb{A})$ stable under the action of $G(\mathbb{A})$ by right translation. If $G=\widetilde{\operatorname{Sp}}(W)$ assume that $\Pi$ consists of genuine functions. Let $u$ be a nilpotent element in $\mathfrak{g}$ such that $\Pi_{N_{u}, \psi_{u}} \neq 0$. Assume that $F J_{\psi_{u}}\left(\Pi_{N_{u}, \psi_{u}}\right)$ (see Section 5 for the definition) is a Whittaker-generic representation of $M(\mathbb{A})$. Then $\Pi_{N_{u^{\prime}}, \psi_{u^{\prime}}} \neq 0$ for a nilpotent element $u^{\prime}$ whose partition is obtained from the partition corresponding to u via replacing a quadruple $(i, i, i, i)$ by $(i+1, i+1, i-1, i-1)$.

\section{SPeCial orbits of Classical GROUPS}

In this section we recall well-known definitions of special orbits and introduce a notion of metaplectic-special orbits for $G=\widetilde{\mathrm{Sp}}(W)$, which has been defined as anti-special in [Mo96].

Definition 10.1. A symplectic orbit is called symplectic special if the number of even parts bigger than every odd $i$ appearing in the corresponding partition is even.

A symplectic orbit is called metaplectic special if the number of even parts bigger than every odd $i$ appearing in the corresponding partition is odd.

An orthogonal orbit is called orthogonal special if the number of odd parts smaller than every even $i$ appearing in the corresponding partition is even. 
Proposition 10.2. A symplectic orbit is symplectic special if the number $m$ given by Lemma 7.1 is even for every odd $i$ appearing in the corresponding partition.

A symplectic orbit is metaplectic special if the number $m$ given by Lemma 7.1 is odd for every odd $i$ appearing in the corresponding partition.

An orthogonal orbit is orthogonal special if the number $m$ given by Lemma 7.1 is even for every even $i$ appearing in the corresponding partition.

Proof. In the first two cases, when $i$ is odd, the parity of $m$ depends on the parity of

$$
\sum_{j>i, j \text { even }} \operatorname{dim} U_{j}
$$

but this number is exactly the number of even parts greater than $i$. In the third case, when $i$ is even, the parity of $m$ depends on the parity of

$$
\sum_{j<i, j \text { odd }} \operatorname{dim} U_{j}
$$

but this number is exactly the number of odd parts less than $i$.

Given a symplectic partition $\underline{p}$ of $2 n$, one easily checks that $p$ is metaplectic special if and only if the transpose of $p$ is an orthogonal partition of $2 n$. Conversely, it is known that an orthogonal partition of $2 n$ is special if and only if its transpose is a symplectic partition. We summarize what we have discussed as the following.

Proposition 10.3. The transpose of partitions defines a bijection between metaplectic-special partitions of $2 n$ and special orthogonal partitions of $2 n$.

Definition 10.4. Given any symplectic partition $p$ of $2 n$, its special expansion $\underline{p}^{\mathrm{Sp}}$ is defined to be the smallest symplectic-special partition which is bigger than $\underline{p}$.

Given any symplectic partition $\underline{p}$ of $2 n$, its metaplectic special expansion $p^{\widetilde{\mathrm{Sp}}}$ is defined to be the smallest metaplectic-special partition of $2 n$ which is bigger than $p$.

Given any orthogonal partition $\underline{p}$ of $m$, its special expansion $\underline{p}^{\mathrm{O}}$ is defined to be the smallest orthogonal-special symplectic partition of $m$ which is bigger than $\underline{p}$.

Lemma 6.3.9 in CM93 gives a recipe for calculating special expansions. In the following proposition, we give a recipe for calculating the metaplectic-expansion. The proof of this proposition is very similar to that of Lemma 6.3.9 in [CM93] and will be omitted.

Proposition 10.5. Let $p=\left[p_{1} p_{2} \cdots p_{r}\right]$ with $p_{1} \geq p_{2} \geq \cdots \geq p_{r}>0$ be a symplectic partition of $2 n$. Let $i_{1}<\cdots<i_{t}$ be the indices $i$ such that $p_{2 i-1}=p_{2 i}$ is odd and $p_{2 i-2} \neq p_{2 i-1}$ or $2 i-2=0$ (the set of indices $\left\{i_{1}, \ldots, i_{t}\right\}$ might be empty). The metaplectic expansion of $\underline{p}$ is obtained by replacing each pair $\left(p_{2 i_{j}-1}, p_{2 i_{j}}\right)$ by $\left(p_{2 i_{j}-1}+1, p_{2 i_{j}}-1\right)$ and leaving the other parts alone.

\section{WAVE-FRONT SETS OF CLASSICAL GROUPS}

Let $G=\operatorname{Sp}(W)$ or $\widetilde{\operatorname{Sp}}(W)$ if $W$ is a symplectic space, or $G=\mathrm{O}(W)$ if $W$ is an orthogonal space. Let $u$ be a nilpotent element in $\mathfrak{g}$, and $p$ the corresponding partition of $\operatorname{dim}(W)$. We shall say that $\underline{p}$ is special if it is metaplectic, symplectic or orthogonal special, respectively. 
Theorem 11.1. Let $k$ be a non-archimedean local field. Let $\pi$ be a smooth representation of $G$. If $G=\widetilde{\operatorname{Sp}}(W)$ assume that $\pi$ is a genuine representation. Let $u$ be a nilpotent element in $\mathfrak{g}$ such that $\pi_{N_{u}, \psi_{u}} \neq 0$. Let $p$ be the partition corresponding to $u$. Then $\pi_{N_{u^{\prime}}, \psi_{u^{\prime}}} \neq 0$ for a nilpotent element $\overline{u^{\prime}}$ whose partition is the special expansion $\underline{p}^{G}$ of $\underline{p}$.

Proof. Assume first that $\pi$ is a genuine representation of $\widetilde{\operatorname{Sp}}(W)$. If the partition $p$ is not metaplectic special then, by Proposition 10.2, there is an odd integer $i$ such that the number $m$ given in Lemma 7.1 is even. So, we are in the situation as in Corollary 6.4 and $F J_{\psi_{u}}\left(\pi_{N_{u}, \psi_{u}}\right)=\operatorname{Hom}_{H_{u, c}}\left(\rho_{\psi_{u}}, \pi_{N_{u}, \psi_{u}}\right)$ is a Whittaker-generic representation of $\mathrm{SL}_{2, \mathfrak{c}}$. Hence by Proposition $\left[8.3, \pi_{N_{u^{\prime}}, \psi_{u^{\prime}}} \neq 0\right.$ for a nilpotent element $u^{\prime}$ whose partition is obtained from the partition of $u$ by replacing a pair $(i, i)$ by $(i+1, i-1)$, as in Proposition 10.5. We can repeat this procedure until the partition is metaplectic special. The cases when $\pi$ is a representation of $\operatorname{Sp}(W)$ or $\mathrm{O}(W)$ are proved analogously. The theorem is proved.

Using Proposition 8.4 (instead of Proposition 8.3) we can prove a global version of Theorem 11.1.

Theorem 11.2. Let $k$ be a global field. Let $\Pi$ be a space of smooth functions on $G(k) \backslash G(\mathbb{A})$ stable under the action of $G(\mathbb{A})$ by right translation. If $G=\widetilde{\mathrm{Sp}}(W)$ assume that $\Pi$ consists of genuine functions. Let $u$ be a nilpotent element in $\mathfrak{g}$ such that $\Pi_{N_{u}, \psi_{u}} \neq 0$. Let $\underline{p}$ be the partition corresponding to $u$. Then $\Pi_{N_{u^{\prime}}, \psi_{u^{\prime}}} \neq 0$ for a nilpotent element $u^{\prime}$ whose partition is the special expansion $\underline{p}^{G}$ of $\underline{p}$.

More refined information about the conjugacy class of $u^{\prime}$ is given by repeated application of Proposition 8.1

\section{WAVE-FRONT SETS OF EXCEPTIONAL GROUPS}

We assume that $G$ is a split, simply-connected exceptional group. Let $(u, s, \bar{u})$ be an $\mathfrak{s l}_{2}$-triple in $\mathfrak{g}$ where $s$ is a semi-simple element. The index of the orbit of $u$ is the value $\kappa(s, s)$ where the Killing form has been normalized so that the index of the orbit corresponding to the long root $\mathfrak{s l}_{2}$ is 1 , as in [Dyn52, Section 10]. For exceptional groups this invariant essentially determines the orbit over a separable closure of $k$. Let $C(s)$ be the centralizer of $s$ in $G$. It is a Levi subgroup of $G$. The centralizer $C$ in $G$ of the $\mathfrak{s l}_{2}$-triple coincides with the stabilizer in $C(s)$ of $v \in \mathfrak{g}(2)$. The absolute type of $C$ is well known, however, a particular choice of $v$ defines a $k$-rational form of $C$.

Let $L$ be the derived group of $C(s)$. It is somewhat easier to work with $L$. We determine the stabilizer $S$ in $L$ of a generic point in $\mathfrak{g}(2)$, on a case-by-case basis, for all non-special orbits using the explicit structure of the $L$-module $\mathfrak{g}(2)$ given in [JN05. Our computation works over most fields. Assuming that the characteristic of $k$ is not a bad prime for $G$ appears to be enough.

Once we have $S$, we check whether the raising conditions in Propositions 6.1 and 6.5 (see also Corollaries 6.4 and 6.6) are met for an $\mathrm{SL}_{2, \mathfrak{c}} \subseteq S$. If so, we enter the corresponding value of $m$ in the table. In all but three cases (Sections 14.3, 17.15 and 17.23) when our method applies, $\mathrm{SL}_{2, c}$ is a long root $\mathrm{SL}_{2}$ in $G$, so conditions (1)-(3) are trivially true and we do not include any additional explanation in these cases. The index of the raised orbit is increased by 1 . 
If our method fails, we consider the method of Mœglin. Now $k$ is a $p$-adic field. Mœglin's result states that if the orbit is not admissible, in the sense of Duflo, then it cannot be a leading term in a wave-front set. The question which orbits of $p$-adic groups are admissible has been studied by Nevins. According to Theorem 3.2 in [N02, the orbit of $u$ is not admissible if there exists $\mathrm{SL}_{2, \mathfrak{c}}$ such that $\mathfrak{g}(1)$, when decomposed as an $\mathrm{SL}_{2, \mathfrak{c}}$-module, satisfies the property that the total number of irreducible summands of dimensions $n \equiv 2(\bmod 4)$ is odd. (In particular, all orbits that satisfy our raising conditions are not admissible.) If an orbit is not admissible and our method fails, we enter $*$ in place of $m$. The remaining orbits are admissible and marked by $* *$. By inspection these orbits are precisely completely odd, non-special orbits. In particular, we have proved that only completely odd orbits are admissible, as conjectured by Nevins.

In the following five sections we tabulate our data. Notation is mostly selfexplanatory, for example, $V_{n}$ denotes the standard representation of $\mathrm{SL}_{n}$ or $\mathrm{Sp}_{n}$, if $n$ is even, or the irreducible $n$-dimensional representation of $\mathrm{SL}_{2}$.

13. $\mathrm{G}_{2}$

\begin{tabular}{|c|cc|c|c|}
\hline \multicolumn{4}{|c|}{ Non-Special Nilpotent Orbits in Type $\mathrm{G}_{2}$} \\
\hline Label & $\begin{array}{c}\text { Diagram } \\
\circ \Rightarrow\end{array}$ & $S$ & $m$ \\
\hline$A_{1}$ & 1 & 0 & $\mathrm{SL}_{2}$ & $* *$ \\
\hline$\widetilde{A}_{1}$ & 0 & 1 & $\mathrm{SL}_{2}$ & 1 \\
\hline
\end{tabular}

13.1. $A_{1}$.

$$
\begin{aligned}
L & =\mathrm{SL}_{2}, \\
\mathfrak{g}(1) & =\operatorname{Sym}^{3} V_{2}=V_{4}, \\
\mathfrak{g}(2) & =V_{1} .
\end{aligned}
$$

Here $S=L$. Neither method works.

13.2. $\widetilde{A}_{1}$.

$$
\begin{aligned}
L & =\mathrm{SL}_{2}, \\
\mathfrak{g}(1) & =V_{2}, \\
\mathfrak{g}(2) & =V_{1} .
\end{aligned}
$$

\begin{tabular}{|c|c|c|c|c|c|c|}
\hline \multicolumn{7}{|c|}{ Non-Special Nilpotent Orbits in Type $\mathrm{F}_{4}$} \\
\hline Label & & $\mathrm{Di}$ & $\mathrm{m}$ & & $S$ & $m$ \\
\hline$A_{1}$ & & & 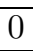 & 0 & $\mathrm{Sp}_{6}$ & 5 \\
\hline$A_{2}+\widetilde{A}_{1}$ & & U & & 0 & $\mathrm{SL}_{2}$ & * \\
\hline$B_{2}$ & 2 & 0 & 0 & 1 & $\mathrm{SL}_{2}(K)$ & 2 \\
\hline$\widetilde{A}_{2}+A_{1}$ & 0 & 1 & 0 & 1 & $\mathrm{SL}_{2}$ & $* *$ \\
\hline$C_{3}\left(a_{1}\right)$ & 1 & 0 & 1 & 0 & $\mathrm{SL}_{2}$ & 3 \\
\hline
\end{tabular}

Here $S=L$ and $m=1$.

14. $\mathrm{F}_{4}$ 
14.1. $A_{1}$.

$$
\begin{aligned}
L & =\mathrm{Sp}_{6}, \\
\mathfrak{g}(1) & =\bigwedge^{3}\left(V_{6}\right) / V_{6}, \\
\mathfrak{g}(2) & =V_{1} .
\end{aligned}
$$

Here $S=L$. Let $\mathrm{SL}_{2, \mathfrak{c}}$ correspond to a long root in $\mathrm{Sp}_{6}$; then $V_{6}=V_{2} \oplus 4 V_{1}$, as an $\mathrm{SL}_{2, \mathfrak{c}}$-module. It follows that $\mathfrak{g}(1)=4 V_{1} \oplus 5 V_{2}$, as an $\mathrm{SL}_{2, \mathfrak{c}}$-module, so $m=5$.

14.2. $A_{2}+\widetilde{A_{1}}$.

$$
\begin{aligned}
L & =\mathrm{SL}_{3} \times \mathrm{SL}_{2}, \\
\mathfrak{g}(1) & =V_{3} \otimes V_{2}, \\
\mathfrak{g}(2) & =V_{3}^{*} \otimes \mathrm{Sym}^{2} V_{2} .
\end{aligned}
$$

Here $\mathfrak{g}(2)$ can be identified with the space of $3 \times 3$ matrices so that the determinant is a relative invariant. The stabilizer $S$ of a generic point is $\mathrm{SL}_{2}$, diagonally embedded in $L$, where $\mathrm{SL}_{2} \rightarrow \mathrm{SL}_{3}$ is given by the natural action of $\mathrm{SL}_{2}$ on $\mathrm{Sym}^{2} V_{2}$. Then $\mathfrak{g}(1)=V_{4} \oplus V_{2}$, as an $\mathrm{SL}_{2, \mathfrak{c}}$-module, only the method of Mœglin works.

14.3. $B_{2}$.

$$
\begin{aligned}
L & =\mathrm{Sp}_{4}, \\
\mathfrak{g}(1) & =V_{4}, \\
\mathfrak{g}(2) & =V_{1} \oplus \bigwedge^{2}\left(V_{4}\right) / V_{1} .
\end{aligned}
$$

Here $V_{5}=\bigwedge^{2}\left(V_{4}\right) / V_{1}$ is the standard representation of $\mathrm{SO}_{5} \cong \mathrm{Sp}_{4} / \mu_{2}$. In particular, there is a degree 2 relative invariant $\Delta$. The stabilizer $S$ of a generic point is $\mathrm{SL}_{2}(K)$ where $K=k(\sqrt{\Delta})$. Let $\mathrm{SL}_{2, \mathfrak{c}}=\mathrm{SL}_{2}(k) \subseteq \mathrm{SL}_{2}(K)$. Then conditions (1)(3) are satisfied with $m=2$. Since 2 is even, Corollaries 6.4 and 6.6 do not apply, i.e., the Fourier-Jacobi model in Propositions 6.1 and 6.5 is not a genuine representation of a 2 -fold central extension of $\mathrm{SL}_{2, \mathfrak{c}}$. However, as an $\mathrm{SL}_{2}(K)$-module, $\mathfrak{g}(1)=V_{2}^{K}$ where $V_{2}^{K}$ is the standard 2-dimensional representation over $K$. In particular, the Fourier-Jacobi model in Propositions 6.1 and 6.5 is a genuine representation of a 2-fold central extension of $\mathrm{SL}_{2}(K)$ and hence it is Whittaker generic as an $\mathrm{SL}_{2, \mathfrak{c}}$-module. Thus the orbit can be raised using Propositions 6.1 and 6.5 .

14.4. $\widetilde{A_{2}}+A_{1}$.

$$
\begin{aligned}
L & =\mathrm{SL}_{2}^{1} \times \mathrm{SL}_{2}^{2}, \\
\mathfrak{g}(1) & =V_{2}^{2} \oplus V_{2}^{1} \otimes \mathrm{Sym}^{2} V_{2}^{2}, \\
\mathfrak{g}(2) & =V_{1} \oplus V_{2}^{1} \otimes V_{2}^{2} .
\end{aligned}
$$

Here $V_{2}^{1} \otimes V_{2}^{2}$ can be identified with the space of $2 \times 2$ matrices so that the determinant is a relative invariant. Hence the stabilizer $S$ of a generic point in $\mathfrak{g}(2)$ is $\mathrm{SL}_{2}$, diagonally embedded in $L$. Then $\mathfrak{g}(1)=2 V_{2} \oplus V_{4}$, as an $\mathrm{SL}_{2, \mathfrak{c}}$-module. Neither method works. 
14.5. $C_{3}\left(a_{1}\right)$.

$$
\begin{aligned}
L & =\mathrm{SL}_{2}^{1} \times \mathrm{SL}_{2}^{2}, \\
\mathfrak{g}(1) & =V_{2}^{1} \oplus V_{2}^{1} \otimes V_{2}^{2}, \\
\mathfrak{g}(2) & =V_{2}^{2} \oplus \operatorname{Sym}^{2} V_{2}^{2} .
\end{aligned}
$$

The space $\mathrm{Sym}^{2} V_{2}^{2}$ has a degree 2 pseudo-invariant. Hence, the stabilizer in $\mathrm{SL}_{2}^{2}$ of a generic point here is $\mathrm{SO}_{2}$. Since the stabilizer in $\mathrm{SO}_{2}$ of a generic point in $V_{2}^{2}$ is trivial, it follows that the stabilizer $S$ of a generic point in $\mathfrak{g}(2)$ is $\mathrm{SL}_{2}^{1}$. Hence,

\begin{tabular}{|c|c|c|c|c|c|c|c|}
\hline \multicolumn{8}{|c|}{ Non-Special Nilpotent Orbits in Type $\mathrm{E}_{6}$} \\
\hline Label & & 0 & $\begin{array}{l}\operatorname{lgr} \\
\circ\end{array}$ & 0 & & $S$ & $m$ \\
\hline $3 A_{1}$ & 0 & 0 & $\begin{array}{l}0 \\
1\end{array}$ & 0 & 0 & $\mathrm{SL}_{3} \times \mathrm{SL}_{2}$ & 9 \\
\hline $2 A_{2}+A_{1}$ & 1 & 0 & $\begin{array}{l}0 \\
1\end{array}$ & 0 & 1 & $\mathrm{SL}_{2}$ & $* *$ \\
\hline$A_{3}+A_{1}$ & 0 & 1 & $\begin{array}{l}1 \\
0\end{array}$ & 1 & 0 & $\mathrm{SL}_{2}$ & 5 \\
\hline$A_{5}$ & 2 & 1 & $\begin{array}{l}1 \\
0\end{array}$ & 1 & 2 & $\mathrm{SL}_{2}$ & 3 \\
\hline
\end{tabular}
$\mathfrak{g}(1)=3 V_{2}$, as an $\mathrm{SL}_{2, \mathfrak{c}}$-module, and $m=3$.

15. $\mathrm{E}_{6}$

15.1. $3 A_{1}$.

$$
\begin{aligned}
L & =\mathrm{SL}_{3}^{1} \times \mathrm{SL}_{2} \times \mathrm{SL}_{3}^{2}, \\
\mathfrak{g}(1) & =V_{3}^{1} \otimes V_{2} \otimes V_{3}^{2, *}, \\
\mathfrak{g}(2) & =V_{3}^{1, *} \otimes V_{3}^{2} .
\end{aligned}
$$

The space $V_{3}^{1, *} \otimes V_{3}^{2}$ can be identified with the spaces of $3 \times 3$ matrices, so that the determinant is a relative invariant. Hence the stabilizer $S$ in $L$ of a generic point in $\mathfrak{g}(2)$ is $\mathrm{SL}_{3} \times \mathrm{SL}_{2}$ where $\mathrm{SL}_{3}$ is diagonally embedded in $\mathrm{SL}_{3}^{1} \times \mathrm{SL}_{3}^{2}$. Let $\mathrm{SL}_{2, \mathfrak{c}}$ be the second factor of $S$. Then $\mathfrak{g}(1)=9 V_{2}$, as an $\mathrm{SL}_{2, \mathfrak{c}}$-module, so $m=9$.

15.2. $2 A_{2}+A_{1}$.

$$
\begin{aligned}
L & =\mathrm{SL}_{2}^{1} \times \mathrm{SL}_{2}^{2} \times \mathrm{SL}_{2}^{3}, \\
\mathfrak{g}(1) & =V_{2}^{1} \oplus V_{2}^{3} \oplus V_{2}^{1} \otimes V_{2}^{2} \otimes V_{2}^{3}, \\
\mathfrak{g}(2) & =V_{1} \oplus V_{2}^{1} \otimes V_{2}^{2} \oplus V_{2}^{2} \otimes V_{2}^{3} .
\end{aligned}
$$

The spaces $V_{2}^{1} \otimes V_{2}^{2}$ and $V_{2}^{2} \otimes V_{2}^{3}$ can be identified with the spaces of $2 \times 2$ matrices. Hence $S=\mathrm{SL}_{2}$, embedded diagonally into the three $\mathrm{SL}_{2}$, and $\mathfrak{g}(1)=4 V_{2} \oplus V_{4}$. Neither method works. 
15.3. $A_{3}+A_{1}$.

$$
\begin{aligned}
L & =\mathrm{SL}_{2}^{1} \times \mathrm{SL}_{2}^{2} \times \mathrm{SL}_{2}^{3}, \\
\mathfrak{g}(1) & =V_{2}^{2} \oplus V_{2}^{1} \otimes V_{2}^{2} \oplus V_{2}^{2} \otimes V_{2}^{3}, \\
\mathfrak{g}(2) & =V_{2}^{1} \oplus V_{2}^{3} \oplus V_{2}^{1} \otimes V_{2}^{3} .
\end{aligned}
$$

The stabilizer in $\mathrm{SL}_{2}^{1} \times \mathrm{SL}_{2}^{3}$ of a generic point in $V_{2}^{1} \otimes V_{2}^{3}$ is $\mathrm{SL}_{2}$, diagonally embedded. The stabilizer in this $\mathrm{SL}_{2}$ of a generic point in $V_{2}^{1} \oplus V_{2}^{3}$ is trivial. Hence $S=\mathrm{SL}_{2}^{2}$ and $\mathfrak{g}(1)=5 V_{2}$, so $m=5$.

15.4. $A_{5}$.

$$
\begin{aligned}
L & =\mathrm{SL}_{2}, \\
\mathfrak{g}(1) & =V_{2} \oplus V_{2} \oplus V_{2}, \\
\mathfrak{g}(2) & =5 V_{1} .
\end{aligned}
$$

\begin{tabular}{|c|c|c|c|c|c|c|c|c|}
\hline \multicolumn{9}{|c|}{ Non-Special Nilpotent Orbits in Type $\mathrm{E}_{7}$} \\
\hline Label & & 0 & $\overline{\mathrm{Di}}$ & $\begin{array}{c}\text { an } \\
0\end{array}$ & 0 & & $S$ & $m$ \\
\hline$\left(3 A_{1}\right)^{\prime}$ & 0 & 0 & 0 & $\begin{array}{l}0 \\
0\end{array}$ & 1 & 0 & $\mathrm{Sp}_{6} \times \mathrm{SL}_{2}$ & 15 \\
\hline $4 A_{1}$ & 1 & 0 & 0 & 0 & 0 & 0 & $\mathrm{Sp}_{6}$ & 7 \\
\hline $2 A_{2}+A_{1}$ & 0 & 1 & 0 & $\begin{array}{l}0 \\
0\end{array}$ & 1 & 0 & $\mathrm{SL}_{2} \times \mathrm{SL}_{2}$ & ** \\
\hline$\left(A_{3}+A_{1}\right)^{\prime}$ & 0 & 0 & 0 & $\begin{array}{l}0 \\
1 \\
\end{array}$ & 0 & 1 & $\mathrm{SL}_{2} \times \mathrm{SL}_{2} \times \mathrm{SL}_{2}$ & 9 \\
\hline$A_{3}+2 A_{1}$ & 1 & 0 & 1 & $\begin{array}{l}0 \\
0\end{array}$ & 0 & 1 & $\mathrm{SL}_{2} \times \mathrm{SL}_{2}$ & 5 \\
\hline$D_{4}+A_{1}$ & 1 & 0 & 0 & $\begin{array}{l}1 \\
0\end{array}$ & 1 & 2 & $\mathrm{Sp}_{4}$ & 3 \\
\hline$\left(A_{5}\right)^{\prime}$ & 0 & 2 & 0 & $\begin{array}{l}0 \\
1\end{array}$ & 0 & 1 & $\mathrm{SL}_{2} \times \mathrm{SL}_{2}$ & 5 \\
\hline$A_{5}+A_{1}$ & 2 & 1 & 0 & $\begin{array}{l}0 \\
1\end{array}$ & 0 & 1 & $\mathrm{SL}_{2}$ & $* *$ \\
\hline$D_{6}\left(a_{2}\right)$ & 2 & 0 & 1 & $\begin{array}{l}1 \\
0\end{array}$ & 1 & 0 & $\mathrm{SL}_{2}$ & 5 \\
\hline$D_{6}$ & 2 & 2 & 1 & 0 & 1 & 2 & $\mathrm{SL}_{2}$ & 3 \\
\hline
\end{tabular}

Here $L=S$. Hence $\mathfrak{g}(1)=3 V_{2}$, so $m=3$.

16. $\mathrm{E}_{7}$ 
16.1. $\left(3 A_{1}\right)^{\prime}$.

$$
\begin{aligned}
L & =\mathrm{SL}_{6} \times \mathrm{SL}_{2}, \\
\mathfrak{g}(1) & =\bigwedge^{2} V_{6} \otimes V_{2}, \\
\mathfrak{g}(2) & =\bigwedge^{2} V_{6}^{*} .
\end{aligned}
$$

The stabilizer $S$ of a generic point in $\mathfrak{g}(2)$ is $\mathrm{Sp}_{6} \times \mathrm{SL}_{2}$. We let $\mathrm{SL}_{2, \mathfrak{c}}$ be the second factor of $S$. Then $\mathfrak{g}(1)=15 V_{2}$, as an $\mathrm{SL}_{2, \mathfrak{c}}$-module, so $m=15$.

16.2. $4 A_{1}$.

$$
\begin{aligned}
L & =\mathrm{SL}_{6}, \\
\mathfrak{g}(1) & =V_{6}^{*} \oplus \bigwedge^{3} V_{6}, \\
\mathfrak{g}(2) & =V_{1} \oplus \bigwedge^{2} V_{6} .
\end{aligned}
$$

The stabilizer $S$ of a generic point in $\mathfrak{g}(2)$ is $\mathrm{Sp}_{6}$. Let $\mathrm{SL}_{2}^{c}$ be a long root $\mathrm{SL}_{2}$. Then $V_{6}=V_{2} \oplus 4 V_{1}$, as an $\mathrm{SL}_{2, \mathfrak{c}}$-module, and $\mathfrak{g}(1)=12 V_{1} \oplus 7 V_{2}$, so $m=7$.

16.3. $2 A_{2}+A_{1}$.

$$
\begin{aligned}
L & =\mathrm{SL}_{2}^{1} \times \mathrm{SL}_{4} \times \mathrm{SL}_{2}^{2}, \\
\mathfrak{g}(1) & =V_{2}^{1} \otimes V_{4}^{*} \oplus \bigwedge^{2} V_{4} \otimes V_{2}^{2}, \\
\mathfrak{g}(2) & =V_{1} \oplus V_{2}^{1} \otimes V_{4} \otimes V_{2}^{2} .
\end{aligned}
$$

The group $\mathrm{SL}_{2}^{1} \times \mathrm{SL}_{2}^{2}$ acting on $V_{2}^{1} \otimes V_{2}^{2}$ gives an identification of $\left(\mathrm{SL}_{2}^{1} \times \mathrm{SL}_{2}^{2}\right) / \mu_{2} \cong$ $\mathrm{SO}_{4}$. Hence the second summand of $\mathfrak{g}(2)$ can be identified with the space of $4 \times 4$ matrices. In this way the determinant is a relative invariant, and the stabilizer $S$ of a generic point of $\mathfrak{g}(2)$ is $\mathrm{SL}_{2} \times \mathrm{SL}_{2}$, embedded diagonally into $L$. It is the tensor product embedding into $\mathrm{SL}_{4}$, so $V_{4}=V_{2}^{1} \otimes V_{2}^{2}$, under the restriction.

Case 1. Let $\mathrm{SL}_{2, \mathfrak{c}}$ be the factor of $S$ isomorphic to $\mathrm{SL}_{2}^{1}$. Then $\mathfrak{g}(1)=8 V_{1} \oplus 4 V_{3}$, as an $\mathrm{SL}_{2, \mathfrak{c}}$-module, hence neither method works.

Case 2. Let $\mathrm{SL}_{2, \mathfrak{c}}$ be the factor of $S$ isomorphic to $\mathrm{SL}_{2}^{2}$. Then $\mathfrak{g}(1)=8 V_{2} \oplus V_{4}$, as an $\mathrm{SL}_{2, \mathfrak{c}}$-module, hence neither method works.

16.4. $\left(A_{3}+A_{1}\right)^{\prime}$.

$$
\begin{aligned}
L & =\mathrm{SL}_{4} \times \mathrm{SL}_{2}^{1} \times \mathrm{SL}_{2}^{2}, \\
\mathfrak{g}(1) & =V_{2}^{2} \oplus V_{4} \otimes V_{2}^{1} \otimes V_{2}^{2}, \\
\mathfrak{g}(2) & =V_{4} \otimes V_{2}^{1} \oplus \bigwedge^{2} V_{4} .
\end{aligned}
$$

Since $\mathrm{SL}_{2}^{2}$ acts trivially on $\mathfrak{g}(2)$, it is a factor of the stabilizer $S$. The stabilizer in $\mathrm{SL}_{4} \times \mathrm{SL}_{2}^{1}$ of a generic point in $\mathfrak{g}(2)$ is $\mathrm{SL}_{2} \times \mathrm{SL}_{2}$. This will be discussed in the next case, as it is not needed here. Let $\mathrm{SL}_{2, \mathfrak{c}}=\mathrm{SL}_{2}^{2}$. Then $\mathfrak{g}(1)=9 V_{2}$, so $m=9$.

16.5. $A_{3}+2 A_{1}$.

$$
\begin{aligned}
L & =\mathrm{SL}_{2} \times \mathrm{SL}_{4}, \\
\mathfrak{g}(1) & =V_{2} \oplus V_{4} \oplus V_{2} \otimes \bigwedge^{2} V_{4}, \\
\mathfrak{g}(2) & =V_{1} \oplus \bigwedge^{2} V_{4} \oplus V_{2} \otimes V_{4}^{*} .
\end{aligned}
$$


A generic element in $V_{2} \otimes V_{4}^{*}$ is contained in $V_{2} \otimes V_{2}^{1}$ where $V_{2}^{1} \subset V_{4}^{*}$ is a 2dimensional subspace. A generic element in $\bigwedge^{2} V_{4}$ is a non-degenerate symplectic form $\omega$ on $V_{4}^{*}$. Let $\mathrm{Sp}_{4} \subset \mathrm{SL}_{4}$ be the stabilizer of $\omega$. Now we have two cases. The restriction of $\omega$ to $V_{2}^{1}$ is trivial or the restriction of $\omega$ to $V_{2}^{1}$ is non-trivial, hence non-degenerate as $\omega$ is a symplectic form. This is the generic case. In this case we can write $V_{4}^{*}=V_{2}^{1} \oplus V_{2}^{2}$ where $V_{2}^{2}$ is the orthogonal complement of $V_{2}^{1}$. Now we have corresponding inclusions

$$
\mathrm{SL}_{2}^{1} \times \mathrm{SL}_{2}^{2} \subset \mathrm{Sp}_{4} \subset \mathrm{SL}_{4}
$$

Hence the stabilizer in $\mathrm{SL}_{2} \times \mathrm{Sp}_{4}$ of a generic point in $V_{2} \otimes V_{2}^{1}$ is $\mathrm{SL}_{2}^{3} \times \mathrm{SL}_{2}^{2}$ where $\mathrm{SL}_{2}^{3}$ is diagonally embedded in $\mathrm{SL}_{2} \times \mathrm{SL}_{2}^{1}$. This is the stabilizer $S$ of a generic point in $\mathfrak{g}(2)$. Let $\mathrm{SL}_{2, \mathfrak{c}}=\mathrm{SL}_{2}^{2}$. Then, as an $\mathrm{SL}_{2, \mathfrak{c}}$-module, $\mathfrak{g}(1)=8 V_{1} \oplus 5 V_{2}$, so $m=5$.

16.6. $D_{4}+A_{1}$.

$$
\begin{aligned}
L & =\mathrm{SL}_{4}, \\
\mathfrak{g}(1) & =2 V_{4} \oplus V_{4}^{*}, \\
\mathfrak{g}(2) & =3 V_{1} \oplus \bigwedge^{2} V_{4} .
\end{aligned}
$$

The stabilizer $S$ of a generic point in $\mathfrak{g}(2)$ is $\mathrm{Sp}_{4}$. Let $\mathrm{SL}_{2, \mathfrak{c}}$ be the long root $\mathrm{SL}_{2}$ in $\mathrm{Sp}_{4}$. Then $V_{4}^{*}=V_{2} \oplus 2 V_{1}$, as an $\mathrm{SL}_{2, \mathfrak{c}}$-module. Hence $\mathfrak{g}(1)=3 V_{2} \oplus 6 V_{1}$, so $m=3$.

16.7. $\left(A_{5}\right)^{\prime}$.

$$
\begin{aligned}
L & =\mathrm{SL}_{2}^{1} \times \mathrm{SL}_{2}^{2} \times \mathrm{SL}_{2}^{3} \times \mathrm{SL}_{2}^{4}, \\
\mathfrak{g}(1) & =V_{2}^{4} \oplus V_{2}^{2} \otimes V_{2}^{3} \otimes V_{2}^{4}, \\
\mathfrak{g}(2) & =V_{1} \oplus V_{2}^{1} \otimes V_{2}^{2} \oplus V_{2}^{2} \otimes V_{2}^{3} .
\end{aligned}
$$

The stabilizer $S$ is $\mathrm{SL}_{2} \times \mathrm{SL}_{2}^{4}$ where $\mathrm{SL}_{2}$ is diagonally embedded into the first three $\mathrm{SL}_{2}$ 's. Let $\mathrm{SL}_{2, \mathfrak{c}}=\mathrm{SL}_{2}^{4}$. Then, as an $\mathrm{SL}_{2, \mathfrak{c}}$-module, $\mathfrak{g}(1)=5 V_{2}^{4}$, so $m=5$.

16.8. $A_{5}+A_{1}$.

$$
\begin{aligned}
L & =\mathrm{SL}_{2}^{1} \times \mathrm{SL}_{2}^{2} \times \mathrm{SL}_{2}^{3}, \\
\mathfrak{g}(1) & =V_{2}^{1} \oplus V_{2}^{3} \oplus V_{2}^{1} \otimes V_{2}^{2} \otimes V_{2}^{3}, \\
\mathfrak{g}(2) & =2 V_{1} \oplus V_{2}^{1} \otimes V_{2}^{2} \oplus V_{2}^{2} \otimes V_{2}^{3} .
\end{aligned}
$$

The same as Section 15.2 .

16.9. $D_{6}\left(a_{2}\right)$.

$$
\begin{aligned}
L & =\mathrm{SL}_{2}^{1} \times \mathrm{SL}_{2}^{2} \times \mathrm{SL}_{2}^{3}, \\
\mathfrak{g}(1) & =V_{2}^{2} \oplus V_{2}^{1} \otimes V_{2}^{2} \oplus V_{2}^{2} \otimes V_{2}^{3}, \\
\mathfrak{g}(2) & =2 V_{2}^{1} \oplus V_{2}^{3} \oplus V_{2}^{1} \otimes V_{2}^{3} .
\end{aligned}
$$

The stabilizer in $\mathrm{SL}_{2}^{1} \times \mathrm{SL}_{2}^{3}$ of a generic point in $V_{2}^{1} \otimes V_{2}^{3}$ is $\mathrm{SL}_{2}$, embedded diagonally. The stabilizer in this $\mathrm{SL}_{2}$ of a generic point in $V_{2}^{1} \oplus V_{2}^{3}$ is trivial. Hence $S=\mathrm{SL}_{2}^{2}$. Then, as an $\mathrm{SL}_{2, \mathfrak{c}}$-module, $\mathfrak{g}(1)=5 V_{2}$, so $m=5$. 
16.10. $D_{6}$.

$$
\begin{aligned}
L & =\mathrm{SL}_{2}, \\
\mathfrak{g}(1) & =V_{2} \oplus V_{2} \oplus V_{2}, \\
\mathfrak{g}(2) & =6 V_{1} .
\end{aligned}
$$

\begin{tabular}{|c|c|c|c|c|c|c|c|c|c|}
\hline \multicolumn{10}{|c|}{ Non-Special Nilpotent Orbits in Type $\mathrm{E}_{8}$} \\
\hline Label & & 0 & 0 & $\lg 1$ & $\begin{array}{l}1 \\
0 \\
0 \\
0\end{array}$ & 0 & & $S$ & $m$ \\
\hline $3 A_{1}$ & 0 & 1 & 0 & 0 & $\begin{array}{l}0 \\
0\end{array}$ & 0 & 0 & $\mathrm{~F}_{4} \times \mathrm{SL}_{2}$ & 27 \\
\hline $4 A_{1}$ & 0 & 0 & 0 & 0 & $\begin{array}{l}1 \\
0\end{array}$ & 0 & 0 & $\mathrm{Sp}_{8}$ & 15 \\
\hline$A_{2}+3 A_{1}$ & 0 & 0 & 0 & 0 & $\begin{array}{l}0 \\
0 \\
\end{array}$ & 1 & 0 & $\mathrm{G}_{2} \times \mathrm{SL}_{2}$ & 21 \\
\hline $2 A_{2}+A_{1}$ & 0 & 1 & 0 & 0 & $\begin{array}{l}0 \\
0\end{array}$ & 0 & 1 & $\mathrm{G}_{2} \times \mathrm{SL}_{2}$ & $* *$ \\
\hline$A_{3}+A_{1}$ & 1 & 0 & 1 & 0 & $\begin{array}{l}0 \\
0\end{array}$ & 0 & 0 & $B_{3} \times \mathrm{SL}_{2}$ & 17 \\
\hline $2 A_{2}+2 A_{1}$ & 0 & 0 & 0 & 1 & $\begin{array}{l}0 \\
0\end{array}$ & 0 & 0 & $\mathrm{Sp}_{4}$ & $* *$ \\
\hline$A_{3}+2 A_{1}$ & 1 & 0 & 0 & 0 & $\begin{array}{l}0 \\
0 \\
\end{array}$ & 1 & 0 & $\mathrm{Sp}_{4} \times \mathrm{SL}_{2}$ & 9 \\
\hline$A_{3}+A_{2}+A_{1}$ & 0 & 0 & 0 & 0 & $\begin{array}{l}0 \\
1\end{array}$ & 0 & 0 & $\mathrm{SL}_{2} \times A_{1}$ & 15 \\
\hline$D_{4}+A_{1}$ & 2 & 1 & 0 & 0 & $\begin{array}{l}1 \\
0\end{array}$ & 0 & 0 & $\mathrm{Sp}_{6}$ & 7 \\
\hline $2 A_{3}$ & 0 & 0 & 0 & 1 & $\begin{array}{l}0 \\
0\end{array}$ & 0 & 1 & $\mathrm{Sp}_{4}$ & $*$ \\
\hline$A_{5}$ & 1 & 0 & 1 & 0 & $\begin{array}{l}0 \\
0\end{array}$ & 0 & 2 & $\mathrm{G}_{2} \times \mathrm{SL}_{2}$ & 9 \\
\hline$A_{4}+A_{3}$ & 0 & 1 & 0 & 0 & $\begin{array}{l}0 \\
1\end{array}$ & 0 & 0 & $\mathrm{SL}_{2}$ & $* *$ \\
\hline$A_{5}+A_{1}$ & 1 & 0 & 0 & 0 & $\begin{array}{l}0 \\
1 \\
\end{array}$ & 0 & 1 & $\mathrm{SL}_{2} \times \mathrm{SL}_{2}$ & 5 \\
\hline$D_{5}\left(a_{1}\right)+A_{2}$ & 1 & 0 & 1 & 0 & $\begin{array}{l}0 \\
0 \\
\end{array}$ & 1 & 0 & $\mathrm{SL}_{2}$ & $*$ \\
\hline$D_{6}\left(a_{2}\right)$ & 0 & 1 & 0 & 0 & $\begin{array}{l}1 \\
0\end{array}$ & 1 & 0 & $\mathrm{SL}_{2}(K)$ & 10 \\
\hline$E_{6}\left(a_{3}\right)+A_{1}$ & 0 & 1 & 0 & 1 & $\begin{array}{l}0 \\
0\end{array}$ & 0 & 1 & $\mathrm{SL}_{2}$ & $* *$ \\
\hline
\end{tabular}

Here $S=L$. Hence $\mathfrak{g}(1)=3 V_{2}$, so $m=3$.

17. $\mathrm{E}_{8}$ 


\begin{tabular}{|c|ccccccc|c|c|}
\hline$E_{7}\left(a_{5}\right)$ & 0 & 0 & 1 & 0 & 1 & 0 & 0 & $\mathrm{SL}_{2} \times \mathrm{Aut}^{1}(E)$ & 9 \\
\hline$D_{5}+A_{1}$ & 2 & 1 & 0 & 1 & 0 & 0 & 1 & $\mathrm{SL}_{2} \times \mathrm{SL}_{2}$ & 5 \\
\hline$D_{6}$ & 2 & 1 & 0 & 0 & 0 & 1 & 2 & $\mathrm{Sp}_{4}$ & 3 \\
\hline$A_{7}$ & 0 & 1 & 1 & 0 & 1 & 0 & 1 & $\mathrm{SL}_{2}$ & $*$ \\
\hline$E_{6}+A_{1}$ & 2 & 2 & 1 & 0 & 1 & 0 & 1 & $\mathrm{SL}_{2}$ & $* *$ \\
\hline$E_{7}\left(a_{2}\right)$ & 2 & 2 & 0 & 1 & 0 & 1 & 0 & $\mathrm{SL}_{2}$ & 5 \\
\hline$D_{7}$ & 1 & 0 & 1 & 1 & 0 & 1 & 2 & $\mathrm{SL}_{2}$ & 5 \\
\hline$E_{7}$ & 2 & 2 & 2 & 1 & 0 & 1 & 2 & $\mathrm{SL}_{2}$ & 3 \\
\hline
\end{tabular}

17.1. $3 A_{1}$.

$$
\begin{aligned}
L & =\mathrm{SL}_{2} \times E_{6}, \\
\mathfrak{g}(1) & =V_{2} \otimes V_{27}^{*}, \\
\mathfrak{g}(2) & =V_{27} .
\end{aligned}
$$

The generic stabilizer $S$ is $\mathrm{SL}_{2} \times F_{4}$. Let $\mathrm{SL}_{2, \mathfrak{c}}=\mathrm{SL}_{2}$, the first factor. Then $\mathfrak{g}(1)=27 V_{2}$, so $m=27$.

17.2. $4 A_{1}$.

$$
\begin{aligned}
L & =\mathrm{SL}_{8}, \\
\mathfrak{g}(1) & =\bigwedge^{3} V_{8}, \\
\mathfrak{g}(2) & =\bigwedge^{2} V_{8}^{*} .
\end{aligned}
$$

The stabilizer $S$ is $\mathrm{Sp}_{8}$. Let $\mathrm{SL}_{2, \mathrm{c}}$ be a long root $\mathrm{SL}_{2}$ in $\mathrm{Sp}_{6}$. Then $V_{8}=V_{2} \oplus 6 V_{1}$, as an $\mathrm{SL}_{2, \mathfrak{c}}$-module. Hence $\mathfrak{g}(1)=26 V_{1} \oplus 15 V_{2}$, so $m=15$.

17.3. $A_{2}+3 A_{1}$.

$$
\begin{aligned}
L & =\mathrm{SL}_{7} \times \mathrm{SL}_{2}, \\
\mathfrak{g}(1) & =\bigwedge^{2} V_{7} \otimes V_{2}, \\
\mathfrak{g}(2) & =\bigwedge^{3} V_{7}^{*} .
\end{aligned}
$$

The stabilizer in $\mathrm{SL}_{7}$ of a generic point in $\bigwedge^{3} V_{7}^{*}$ is $G_{2}$. Hence the stabilizer $S$ is $G_{2} \times \mathrm{SL}_{2}$. Let $\mathrm{SL}_{2, \mathfrak{c}}=\mathrm{SL}_{2}$, the second factor in $S$. Then, as an $\mathrm{SL}_{2, \mathfrak{c}}$-module, $\mathfrak{g}(1)=21 V_{2}$, so $m=21$.

17.4. $2 A_{2}+A_{1}$.

$$
\begin{aligned}
L & =\mathrm{SL}_{2} \times \operatorname{Spin}_{10}, \\
\mathfrak{g}(1) & =V_{2} \otimes V_{10} \oplus V_{16}^{2}, \\
\mathfrak{g}(2) & =V_{1} \oplus V_{2} \otimes V_{16}^{1} .
\end{aligned}
$$


Here $V_{16}^{i}(i=1,2)$ denotes two inequivalent spin representations. A generic element in $V_{2} \otimes V_{16}^{1}$ is contained in $V_{2} \otimes V_{2}^{1}$ for some 2-dimensional subspace $V_{2}^{1} \subset V_{16}^{1}$. The point-wise stabilizer in $\operatorname{Spin}_{10}$ of a generic $V_{2}^{1}$ is $G_{2}$. The centralizer of $G_{2}$ in $\operatorname{Spin}_{10}$ is $\operatorname{Spin}_{3}$. Since $\operatorname{Spin}_{3}$ acts on $V_{2}^{1}$, we have an identification $\operatorname{Spin}_{3} \cong \mathrm{SL}_{2}^{1}$. It follows that the stabilizer $S$ of a generic point in $\mathfrak{g}(2)$ is $\mathrm{SL}_{2} \times G_{2}$ where $\mathrm{SL}_{2}$ is embedded diagonally.

Case 1. Let $\mathrm{SL}_{2, \mathrm{c}}$ be the first factor of $S$. Then $V_{10}=V_{3} \oplus 7 V_{1}$ and $V_{16}^{2}=8 V_{2}$ as $\mathrm{SL}_{2, \mathfrak{c}}$-modules. Then $\mathfrak{g}(1)=16 V_{2} \oplus V_{4}$, hence neither method works.

Case 2. Take the $\mathrm{SL}_{2, \mathfrak{c}}$ corresponding to a long root $\mathrm{SL}_{2}$ in $G_{2}$. It is a root $\mathrm{SL}_{2}$ in $\operatorname{Spin}_{10}$. Then $V_{10}=2 V_{2} \oplus 6 V_{1}$ and $V_{16}=4 V_{3} \oplus 8 V_{1}$, as $\mathrm{SL}_{2, \mathfrak{c}}$-modules, and $\mathfrak{g}(1)=20 V_{1} \oplus 8 V_{2}$. Hence neither method works.

17.5. $A_{3}+A_{1}$.

$$
\begin{aligned}
L & =\mathrm{SL}_{2} \times \operatorname{Spin}_{10}, \\
\mathfrak{g}(1) & =V_{2} \oplus V_{2} \otimes V_{16}, \\
\mathfrak{g}(2) & =V_{16} \oplus V_{10} .
\end{aligned}
$$

Here $V_{16}$ denotes a spin representation. Note that $\mathrm{SL}_{2}$, the first factor of $L$, is always in $S$. So we let $\mathrm{SL}_{2, \mathfrak{c}}$ be this $\mathrm{SL}_{2}$. Then $\mathfrak{g}(1)=17 V_{2}$, as an $\mathrm{SL}_{2, \mathfrak{c}}$-module, so $m=17$.

17.6. $2 A_{2}+2 A_{1}$.

$$
\begin{aligned}
L & =\mathrm{SL}_{4} \times \mathrm{SL}_{5}, \\
\mathfrak{g}(1) & =V_{4} \otimes \bigwedge^{2} V_{5}^{*}, \\
\mathfrak{g}(2) & =\bigwedge^{2} V_{4} \otimes V_{5} .
\end{aligned}
$$

Write $V_{6}=\bigwedge^{2} V_{4}$. The action of $\mathrm{SL}_{4}$ on $V_{6}$ gives an isomorphism $\mathrm{SL}_{4} / \mu_{2} \cong \mathrm{SO}_{6}$. A generic element in $V_{6} \otimes V_{5}$ is contained in $V_{5}^{1} \otimes V_{5}$ for a 5 -dimensional non-degenerate subspace $V_{5}^{1} \subset V_{6}$. Note that $V_{5}^{1}$ is a split quadratic space, as $V_{6}$ is. The stabilizer $S$ of a generic point is $\mathrm{Sp}_{4} \cong \operatorname{Spin}\left(V_{5}^{1}\right)$ embedded diagonally in $\mathrm{SL}_{4} \times \mathrm{SL}_{5}$. Let $\mathrm{SL}_{2, \mathfrak{c}}$ be a long root $\mathrm{SL}_{2}$ in $\mathrm{Sp}_{4}$. Then $V_{4}=V_{2} \oplus 2 V_{1}$ and $V_{5}=2 V_{2} \oplus V_{1}$, as $\mathrm{SL}_{2, c}$-modules, and $\mathfrak{g}(1)=8 V_{1} \oplus 8 V_{2} \oplus 4 V_{3} \oplus V_{4}$. Hence neither method works.

17.7. $A_{3}+2 A_{1}$.

$$
\begin{aligned}
L & =\mathrm{SL}_{6} \times \mathrm{SL}_{2}, \\
\mathfrak{g}(1) & =V_{6}^{*} \oplus \bigwedge^{2} V_{6} \otimes V_{2}, \\
\mathfrak{g}(2) & =V_{6} \otimes V_{2} \oplus \bigwedge^{2} V_{6}^{*} .
\end{aligned}
$$

A generic element in $\bigwedge^{2} V_{6}^{*}$ is a non-degenerate symplectic form $\omega$ on $V_{6}$. Let $\mathrm{Sp}_{6}$ be the stabilizer of $\omega$ in $\mathrm{SL}_{6}$. A generic element in $V_{6} \otimes V_{2}$ is contained in $V_{2}^{1} \otimes V_{2}$ where $V_{2}^{1}$ is a 2-dimensional subspace of $V_{6}$. We are in a generic situation when $\omega$ restricts to a non-trivial form on $V_{2}^{1}$. In this case we can decompose $V_{6}=V_{2}^{1} \oplus V_{4}$. This gives an embedding $\mathrm{SL}_{2}^{1} \times \mathrm{Sp}_{4} \subset \mathrm{Sp}_{6}$. The centralizer $S$ of a generic element in $\mathfrak{g}(2)$ is $\mathrm{Sp}_{4} \times \mathrm{SL}_{2}$ where $\mathrm{SL}_{2}$ is diagonally embedded in $\mathrm{SL}_{2}^{1}$ and the second factor of $L$. Let $\mathrm{SL}_{2, \mathfrak{c}}$ be a long root $\mathrm{SL}_{2}$ in $\mathrm{Sp}_{4}$. Then $V_{6}^{*} \cong V_{6}=V_{2} \oplus 4 V_{1}$, as $\mathrm{SL}_{2, \mathfrak{c}}$-modules, and $\mathfrak{g}(1)=18 V_{1} \oplus 9 V_{2}$, so $m=9$. 
17.8. $A_{3}+A_{2}+A_{1}$.

$$
\begin{aligned}
L & =\mathrm{SL}_{5} \times \mathrm{SL}_{2} \times \mathrm{SL}_{3}, \\
\mathfrak{g}(1) & =V_{5} \otimes V_{2} \otimes V_{3}^{*}, \\
\mathfrak{g}(2) & =\bigwedge^{2} V_{5} \otimes V_{3} .
\end{aligned}
$$

We take $\mathrm{SL}_{2, \mathfrak{c}}$ to be the second factor of $L$; it is clearly contained in $S$. Then $\mathfrak{g}(1)=15 V_{2}$, as an $\mathrm{SL}_{2, \mathfrak{c}}$-module, so $m=15$.

17.9. $D_{4}+A_{1}$.

$$
\begin{aligned}
L & =\mathrm{SL}_{6}, \\
\mathfrak{g}(1) & =V_{6}^{*} \oplus \bigwedge^{3} V_{6}, \\
\mathfrak{g}(2) & =2 V_{1} \oplus \bigwedge^{2} V_{6} .
\end{aligned}
$$

The same as Section 16.2.

17.10. $2 A_{3}$.

$$
\begin{aligned}
L & =\mathrm{SL}_{4}^{1} \times \mathrm{SL}_{4}^{2}, \\
\mathfrak{g}(1) & =V_{4}^{2} \oplus V_{4}^{1} \otimes \bigwedge^{2} V_{4}^{2}, \\
\mathfrak{g}(2) & =V_{4}^{1} \otimes V_{4}^{2, *} \oplus \bigwedge^{2} V_{4}^{1} .
\end{aligned}
$$

The stabilizer of a generic point in $V_{4}^{1} \otimes V_{4}^{2, *}$ is $\mathrm{SL}_{4}$, embedded diagonally. The stabilizer of a generic point in $\bigwedge^{2} V_{4}^{1}$ is $\mathrm{Sp}_{4} \times \mathrm{SL}_{4}^{2}$. Hence $S=\mathrm{Sp}_{4}$, embedded diagonally. Let $\mathrm{SL}_{2, \mathfrak{c}}$ be a long root $\mathrm{SL}_{2}$ in $\mathrm{Sp}_{4}$. Then, as $\mathrm{SL}_{2}^{c}$-modules, $V_{4}^{1}=V_{4}^{2}=$ $V_{2}+2 V_{1}$. Hence $\mathfrak{g}(1)=8 V_{1} \oplus 7 V_{2} \oplus 2 V_{3}$ and the method of Mœeglin works.

17.11. $A_{5}$.

$$
\begin{aligned}
L & =\mathrm{SL}_{2} \times \operatorname{Spin}_{8}, \\
\mathfrak{g}(1) & =V_{2} \oplus V_{2} \otimes V_{8}, \\
\mathfrak{g}(2) & =V_{8} \oplus V_{1} \oplus V_{8}^{\prime} .
\end{aligned}
$$

Here we can proclaim $V_{8}$ in $\mathfrak{g}(1)$ be the standard representation; then $V_{8}^{\prime}$ is a spin representation. Note that $\mathrm{SL}_{2}$, the first factor of $L$, is always in $S$. So we let $\mathrm{SL}_{2, \mathfrak{c}}$ be this $\mathrm{SL}_{2}$. Then $\mathfrak{g}(1)=9 V_{2}$, as an $\mathrm{SL}_{2, \mathfrak{c}}$-module, so $m=9$.

17.12. $A_{4}+A_{3}$.

$$
\begin{aligned}
L & =\mathrm{SL}_{2}^{1} \times \mathrm{SL}_{3}^{1} \times \mathrm{SL}_{2}^{2} \times \mathrm{SL}_{3}^{2}, \\
\mathfrak{g}(1) & =V_{2}^{1} \otimes V_{3}^{1, *} \oplus V_{3}^{1} \otimes V_{2}^{2} \otimes V_{3}^{2, *}, \\
\mathfrak{g}(2) & =V_{2}^{1} \otimes V_{2}^{2} \otimes V_{3}^{2, *} \oplus V_{3}^{1, *} \otimes V_{3}^{2} .
\end{aligned}
$$

The stabilizer is $\mathrm{SL}_{2}$, diagonally embedded into all factors of $L$, where we use the symmetric square embedding into two $\mathrm{SL}_{3}$ 's. Then $\mathfrak{g}(1)=3 V_{2} \oplus 3 V_{4} \oplus V_{6}$ and neither method works. 
17.13. $A_{5}+A_{1}$.

$$
\begin{aligned}
L & =\mathrm{SL}_{4} \times \mathrm{SL}_{2}^{1} \times \mathrm{SL}_{2}^{2}, \\
\mathfrak{g}(1) & =V_{2}^{2} \oplus V_{4}^{*} \oplus V_{4} \otimes V_{2}^{1} \otimes V_{2}^{2}, \\
\mathfrak{g}(2) & =V_{4} \otimes V_{2}^{1} \oplus V_{2}^{1} \otimes V_{2}^{2} \oplus \bigwedge^{2} V_{4} .
\end{aligned}
$$

The stabilizer of a generic element in $V_{2}^{1} \otimes V_{2}^{2}$ is $\mathrm{SL}_{2}$, diagonally embedded into the last two factors of $L$. The stabilizer in $\mathrm{SL}_{4}$ of a generic element in $\bigwedge^{2} V_{4}$ is $\mathrm{Sp}_{4}$. As in Section 16.5, one can write $V_{4}=V_{2}^{3} \oplus V_{2}^{4}$, such that the stabilizer in $\mathrm{Sp}_{4} \times \mathrm{SL}_{2}^{1}$ of a generic element in $V_{4} \otimes V_{2}^{1}$ is $\mathrm{SL}_{2}^{3} \times \mathrm{SL}_{2}$, where the second $\mathrm{SL}_{2}$ is diagonally embedded to $\mathrm{SL}_{2}^{4}$ and $\mathrm{SL}_{2}^{1}$. Hence $S=\mathrm{SL}_{2}^{3} \times \mathrm{SL}_{2}$, where the second $\mathrm{SL}_{2}$ is diagonally embedded in $\mathrm{SL}_{2}^{4}, \mathrm{SL}_{2}^{1}$ and $\mathrm{SL}_{2}^{2}$. Let $\mathrm{SL}_{2, \mathfrak{c}}=\mathrm{SL}_{2}^{3}$. Then $\mathfrak{g}(1)=12 V_{1} \oplus 5 V_{2}$, so $m=5$.

17.14. $D_{5}\left(a_{1}\right)+A_{2}$.

$$
\begin{aligned}
L & =\mathrm{SL}_{2}^{1} \times \mathrm{SL}_{4} \times \mathrm{SL}_{2}^{2}, \\
\mathfrak{g}(1) & =V_{2}^{1} \oplus V_{2}^{1} \otimes V_{4}^{*} \oplus \bigwedge^{2} V_{4} \otimes V_{2}^{2}, \\
\mathfrak{g}(2) & =V_{1} \oplus V_{4}^{*} \oplus V_{2}^{1} \otimes V_{4} \otimes V_{2}^{2} .
\end{aligned}
$$

As in Section 16.3, the stabilizer of a generic element in $V_{2}^{1} \otimes V_{4} \otimes V_{2}^{2}$ is $\mathrm{SL}_{2} \times \mathrm{SL}_{2}$, diagonally embedded in $L$, where embedding into $\mathrm{SL}_{4}$ is given by the tensor product. The stabilizer in $\mathrm{SL}_{2} \times \mathrm{SL}_{2}$ of a generic element in $V_{4}^{*}$ is $\mathrm{SL}_{2}$, diagonally embedded. Hence, $S$ is $\mathrm{SL}_{2}$ and, as an $\mathrm{SL}_{2, \mathfrak{c}}$-module, $\mathfrak{g}(1)=3 V_{2} \oplus 2 V_{3} \oplus V_{4}$. Hence, only the method of Mœeglin works.

17.15. $D_{6}\left(a_{2}\right)$.

$$
\begin{aligned}
L & =\mathrm{SL}_{2}^{1} \times \mathrm{SL}_{4} \times \mathrm{SL}_{2}^{2}, \\
\mathfrak{g}(1) & =V_{4} \oplus V_{2}^{1} \otimes V_{4}^{*} \oplus V_{4} \otimes V_{2}^{2}, \\
\mathfrak{g}(2) & =V_{2}^{1} \oplus V_{2}^{1} \otimes V_{2}^{2} \oplus \bigwedge^{2} V_{4} \otimes V_{2}^{2} .
\end{aligned}
$$

The stabilizer in $\mathrm{SL}_{2}^{1} \times \mathrm{SL}_{2}^{2}$ of a generic element in $V_{2}^{1} \otimes V_{2}^{2}$ is $\mathrm{SL}_{2}$, diagonally embedded. The action of $\mathrm{SL}_{4}$ on $\bigwedge^{2} V_{4}$ gives an isomorphism $\mathrm{SL}_{4} / \mu_{2} \cong \mathrm{SO}_{6}$. A generic element in $\bigwedge^{2} V_{4} \otimes V_{2}^{2}$ is contained in $V_{2}^{3} \otimes V_{2}^{2}$ where $V_{2}^{3} \subset \bigwedge^{2} V_{4}$ is a nondegenerate 2-dimensional quadratic subspace. Write $\bigwedge^{2} V_{4}=V_{2}^{3} \oplus V_{4}^{3}$. Hence the stabilizer in $\mathrm{SO}_{4} \times \mathrm{SL}_{2}^{2}$ of a generic element in $\bigwedge^{2} V_{4} \otimes V_{2}^{2}$ is $\mathrm{SO}_{2} \times \mathrm{SO}\left(V_{4}^{3}\right)$ where $\mathrm{SO}_{2}$ is diagonally embedded in $\mathrm{SO}\left(V_{2}^{3}\right)$ and $\mathrm{SO}_{2} \subset \mathrm{SL}_{2}^{2}$. Next, the stabilizer in $\mathrm{SL}_{2}^{1} \times \mathrm{SL}_{2}^{2}$ of a generic element in $V_{2}^{1} \otimes V_{2}^{2}$ is $\mathrm{SL}_{2}$ diagonally embedded. Furthermore, the stabilizer in $\mathrm{SO}_{2}$ of a generic point in $V_{2}^{1}$ is trivial. Hence the stabilizer $S$ of a generic point in $\mathfrak{g}(2)$ is $\operatorname{Spin}\left(V_{4}^{3}\right) \cong \mathrm{SL}_{2}(K) \subset \mathrm{SL}_{4}$, where $K$ is a quadratic algebra. Let $\mathrm{SL}_{2, \mathfrak{c}}=\mathrm{SL}_{2}(k) \subseteq \mathrm{SL}_{2}(K)$. Then conditions (1)-(3) are satisfied with $m=10$. Since 10 is even, Corollaries 6.4 and 6.6 do not apply, i.e., the FourierJacobi model in Propositions 6.1 and 6.5 is not a genuine representation of a 2-fold central extension of $\mathrm{SL}_{2, \mathfrak{c}}$. However, as an $\mathrm{SL}_{2}(K)$-module, $\mathfrak{g}(1)=5 V_{2}^{K}$ where $V_{2}^{K}$ is the standard 2-dimensional representation over $K$. In particular, the FourierJacobi model in Propositions 6.1 and 6.5 is a genuine representation of a 2-fold central extension of $\mathrm{SL}_{2}(K)$ and hence it is Whittaker generic as an $\mathrm{SL}_{2, \mathfrak{c}}$-module. Thus the orbit can be raised using Propositions 6.1 and 6.5. 
17.16. $E_{6}\left(a_{3}\right)+A_{1}$.

$$
\begin{aligned}
L & =\mathrm{SL}_{2}^{1} \times \mathrm{SL}_{2}^{2} \times \mathrm{SL}_{4}, \\
\mathfrak{g}(1) & =V_{2}^{1} \otimes V_{2}^{2} \oplus V_{4} \oplus V_{2}^{2} \otimes \bigwedge^{2} V_{4}, \\
\mathfrak{g}(2) & =V_{1} \oplus V_{2}^{2} \otimes V_{4}^{*} \oplus V_{2}^{1} \otimes \bigwedge^{2} V_{4} .
\end{aligned}
$$

This is somewhat similar to the previous case, so let $\mathrm{SO}_{2} \times \mathrm{SO}\left(V_{4}^{3}\right)$ be the stabilizer in $\mathrm{SL}_{2}^{1} \times \mathrm{SO}_{6}$. Ignoring the factor $\mathrm{SO}_{2}$, we must compute the stabilizer in $\mathrm{SL}_{2}^{2} \times$ $\mathrm{SL}_{2}(K)$ of a generic point in $V_{2}^{2} \otimes V_{4}^{*}$. It is $\mathrm{SL}_{2}$ diagonally embedded into $\mathrm{SL}_{2}^{2} \times$ $\mathrm{SL}_{2}(K)$. (This is evident when $K=k \oplus k$, and by Galois descent in general.) Hence $\mathrm{SL}_{2, \mathfrak{c}}$ is embedded into $\mathrm{SL}_{2}^{2}$ and $\mathrm{SL}_{4}$, so that $V_{4}=2 V_{2}$, as an $\mathrm{SL}_{2, \mathfrak{c}}$-module. Hence $\mathfrak{g}(1)=8 V_{2} \oplus V_{4}$. Hence neither method works.

17.17. $E_{7}\left(a_{5}\right)$.

$$
\begin{aligned}
L & =\mathrm{SL}_{3}^{1} \times \mathrm{SL}_{2}^{1} \times \mathrm{SL}_{2}^{2} \times \mathrm{SL}_{3}^{2}, \\
\mathfrak{g}(1) & =V_{3}^{1} \otimes V_{2}^{1} \oplus V_{2}^{1} \otimes V_{2}^{2} \otimes V_{3}^{2, *}, \\
\mathfrak{g}(2) & =V_{3}^{2} \oplus V_{3}^{1} \otimes V_{2}^{2} \otimes V_{3}^{2, *} .
\end{aligned}
$$

It is clear that $\mathrm{SL}_{2}^{1}$ is always in $S$. (The 18-dimensional summand in $\mathfrak{g}(2)$ is the Bhargava $3 \times 2 \times 3$ cube. A generic cube corresponds to a cubic separable algebra $E$ over $k$, and the stabilizer in $\mathrm{SL}_{3}^{1} \times \mathrm{SL}_{2}^{2} \times \mathrm{SL}_{3}^{2}$ is isomorphic to $E^{1} \rtimes \operatorname{Aut}^{1}(E)$ where $E^{1}$ is the torus of norm one elements in $E^{\times}$and $\operatorname{Aut}^{1}(E)$ is the group of $k$ automorphisms of $E$ of determinant 1.) Let $\mathrm{SL}_{2, \mathfrak{c}}=\mathrm{SL}_{2}^{1}$. Then, as an $\mathrm{SL}_{2, \mathfrak{c}}$-module, $\mathfrak{g}(1)=9 V_{2}^{1}$. Hence, $m=9$.

17.18. $D_{5}+A_{1}$.

$$
\begin{aligned}
L & =\mathrm{SL}_{2} \times \mathrm{SL}_{4}, \\
\mathfrak{g}(1) & =V_{2} \oplus V_{4} \oplus V_{2} \otimes \bigwedge^{2} V_{4}, \\
\mathfrak{g}(2) & =2 V_{1} \oplus \bigwedge^{2} V_{4} \oplus V_{2} \otimes V_{4}^{*} .
\end{aligned}
$$

The same as Section 16.5.

17.19. $D_{6}$.

$$
\begin{aligned}
L & =\mathrm{SL}_{4}, \\
\mathfrak{g}(1) & =V_{4}^{*} \oplus 2 V_{4}, \\
\mathfrak{g}(2) & =4 V_{1} \oplus \bigwedge^{2} V_{4} .
\end{aligned}
$$

The same as Section 16.6 .

17.20. $A_{7}$.

$$
\begin{aligned}
L & =\mathrm{SL}_{2}^{1} \times \mathrm{SL}_{2}^{2} \times \mathrm{SL}_{2}^{3} \times \mathrm{SL}_{2}^{4}, \\
\mathfrak{g}(1) & =V_{2}^{1} \oplus V_{2}^{2} \oplus V_{2}^{4} \oplus V_{2}^{2} \otimes V_{2}^{3} \otimes V_{2}^{4}, \\
\mathfrak{g}(2) & =V_{1} \oplus V_{2}^{1} \otimes V_{2}^{2} \oplus V_{2}^{2} \otimes V_{2}^{3} \oplus V_{2}^{3} \otimes V_{2}^{4} .
\end{aligned}
$$

The stabilizer $S$ of a generic point in $\mathfrak{g}(2)$ is $\mathrm{SL}_{2}$ embedded diagonally in the four $\mathrm{SL}_{2}$. Hence $\mathfrak{g}(1)=5 V_{2} \oplus V_{4}$, and the method of Mœglin works. 
17.21. $E_{6}+A_{1}$.

$$
\begin{aligned}
L & =\mathrm{SL}_{2}^{1} \times \mathrm{SL}_{2}^{2} \times \mathrm{SL}_{2}^{3}, \\
\mathfrak{g}(1) & =V_{2}^{3} \oplus V_{2}^{1} \oplus V_{2}^{1} \otimes V_{2}^{2} \otimes V_{2}^{3}, \\
\mathfrak{g}(2) & =3 V_{1} \oplus V_{2}^{1} \otimes V_{2}^{2} \oplus V_{2}^{2} \otimes V_{2}^{3} .
\end{aligned}
$$

The same as Section 15.2

17.22. $E_{7}\left(a_{2}\right)$.

$$
\begin{aligned}
L & =\mathrm{SL}_{2}^{1} \times \mathrm{SL}_{2}^{2} \times \mathrm{SL}_{2}^{3}, \\
\mathfrak{g}(1) & =V_{2}^{2} \oplus V_{2}^{1} \otimes V_{2}^{2} \oplus V_{2}^{2} \otimes V_{2}^{3}, \\
\mathfrak{g}(2) & =V_{1} \oplus 2 V_{2}^{1} \oplus V_{2}^{3} \oplus V_{2}^{1} \otimes V_{2}^{3} .
\end{aligned}
$$

The same as Section 16.9

17.23. $D_{7}$.

$$
\begin{aligned}
L & =\mathrm{SL}_{2}^{1} \times \mathrm{SL}_{2}^{2}, \\
\mathfrak{g}(1) & =2 V_{2}^{1} \oplus 3 V_{2}^{2}, \\
\mathfrak{g}(2) & =5 V_{1} \oplus V_{2}^{1} \otimes V_{2}^{2}, \\
\mathfrak{g}(3) & =2 V_{2}^{1} \oplus 3 V_{2}^{2}, \\
\mathfrak{g}(4) & =4 V_{1} \oplus V_{2}^{1} \otimes V_{2}^{2}, \\
\mathfrak{g}(5) & =2 V_{2}^{1} \oplus 2 V_{2}^{2} .
\end{aligned}
$$

The stabilizer $S$ of a generic point in $\mathfrak{g}(2)$ is $\mathrm{SL}_{2}$ embedded diagonally in the two $\mathrm{SL}_{2}$. Hence $\mathfrak{g}(1)=5 V_{2}$, so $m=5$. Note that $\operatorname{dim} \mathfrak{g}(0,2)=2$ and $\operatorname{dim} \mathfrak{g}(2,2)=1$, and the $\varphi_{\mathfrak{c}}$-weights are bounded by 2 .

17.24. $E_{7}$.

$$
\begin{aligned}
L & =\mathrm{SL}_{2}, \\
\mathfrak{g}(1) & =V_{2} \oplus V_{2} \oplus V_{2}, \\
\mathfrak{g}(2) & =7 V_{1} .
\end{aligned}
$$

Here $S=L$. Hence $\mathfrak{g}(1)=3 V_{2}$, so $m=3$.

\section{ACKNOWLEDGMENTS}

The authors would like to thank Peter Trapa for a crystalizing conversation concerning the definition of metaplectic special orbits, Joseph Hundley for pointing out the paper [N02, and Monica Nevins for a correspondence on that paper. A part of this paper was written during the program on New Geometric Methods in Number Theory at MSRI, Berkeley. The authors have been partially supported by grants from NSF, DMS-1600685, DMS-1302122, DMS-1620329 and DMS-1359774, respectively. The second-named author was also supported by start-up funds from the Department of Mathematics, Purdue University. The authors also would like to thank the referee for helpful comments and suggestions.

This material is based upon work supported by the National Science Foundation under agreement No. DMS-1128155. Any opinions, findings and conclusions or recommendations expressed in this material are those of the authors and do not necessarily reflect the views of the National Science Foundation. 


\section{REFERENCES}

[BS98] Rolf Berndt and Ralf Schmidt, Elements of the representation theory of the Jacobi group, Progress in Mathematics, vol. 163, Birkhäuser Verlag, Basel, 1998. MR.1634977

[Ca93] Roger W. Carter, Finite groups of Lie type: Conjugacy classes and complex characters, Wiley Classics Library, John Wiley \& Sons, Ltd., Chichester, 1993. Reprint of the 1985 original; A Wiley-Interscience Publication. MR.1266626

[CM93] David H. Collingwood and William M. McGovern, Nilpotent orbits in semisimple Lie algebras, Van Nostrand Reinhold Mathematics Series, Van Nostrand Reinhold Co., New York, 1993. MR:1251060

[Dyn52] E. B. Dynkin, Semisimple subalgebras of semisimple Lie algebras (Russian), Mat. Sbornik N.S. 30(72) (1952), 349-462 (3 plates). MR0047629

[G06] David Ginzburg, Certain conjectures relating unipotent orbits to automorphic representations, Israel J. Math. 151 (2006), 323-355, DOI 10.1007/BF02777366. MR2214128

[GRS03] D. Ginzburg, S. Rallis, and D. Soudry, On Fourier coefficients of automorphic forms of symplectic groups, Manuscripta Math. 111 (2003), no. 1, 1-16, DOI 10.1007/s00229003-0355-7. MR 1981592

[JN05] Steven Glenn Jackson and Alfred G. Noël, Prehomogeneous spaces associated with complex nilpotent orbits, J. Algebra 289 (2005), no. 2, 515-557, DOI 10.1016/j.jalgebra.2005.02.017. MR2142384

[JL15] Dihua Jiang and Baiying Liu, On special unipotent orbits and Fourier coefficients for automorphic forms on symplectic groups, J. Number Theory 146 (2015), 343-389, DOI 10.1016/j.jnt.2014.03.002. MR3267118

[LS08] Hung Yean Loke and Gordan Savin, On minimal representations of Chevalley groups of type $D_{n}, E_{n}$ and $G_{2}$, Math. Ann. 340 (2008), no. 1, 195-208, DOI 10.1007/s00208-0070144-9. MR 2349773

[Mo96] C. Mœglin, Front d'onde des représentations des groupes classiques p-adiques (French, with French summary), Amer. J. Math. 118 (1996), no. 6, 1313-1346. MR.1420926

[MW87] C. Møglin and J.-L. Waldspurger, Modèles de Whittaker dégénérés pour des groupes p-adiques (French), Math. Z. 196 (1987), no. 3, 427-452, DOI 10.1007/BF01200363. MR.913667

[MW95] C. Mœglin and J.-L. Waldspurger, Spectral decomposition and Eisenstein series, Cambridge Tracts in Mathematics, vol. 113, Cambridge University Press, Cambridge, 1995. MR 1361168

[N02] Monica Nevins, Admissible nilpotent orbits of real and p-adic split exceptional groups, Represent. Theory 6 (2002), 160-189 (electronic), DOI 10.1090/S1088-4165-02-00134-6. MR 1915090

[V14] Sandeep Varma, On a result of Moeglin and Waldspurger in residual characteristic 2, Math. Z. 277 (2014), no. 3-4, 1027-1048, DOI 10.1007/s00209-014-1292-8. MR3229979

[Wal01] Jean-Loup Waldspurger, Intégrales orbitales nilpotentes et endoscopie pour les groupes classiques non ramifiés (French, with English and French summaries), Astérisque 269 (2001), vi+449. MR 1817880

[Wei03] Martin H. Weissman, The Fourier-Jacobi map and small representations, Represent. Theory 7 (2003), 275-299 (electronic), DOI 10.1090/S1088-4165-03-00197-3. MR.1993361

School of Mathematics, University of Minnesota, Minneapolis, Minnesota 55455

E-mail address: dhjiang@math.umn.edu

Department of Mathematics, Purdue University, 150 N. University Street, West LAFAYETTE, INDIANA 47907

E-mail address: liu2053@purdue.edu

Department of Mathematics, University of Utah, Salt Lake City, Utah 84112

E-mail address: savin@math.utah.edu 